ORNL/TM--12211

DE93 005262

\title{
Reducing Emissions from Uranium Dissolving
}

\author{
W. L. Griffith \\ A. L. Compere \\ W. P. Huxtable \\ J. M. Googin
}
Prepared by the Oak Ridge National Laboratory
Oak Ridge, Tennessee 37831 managed by
Martin Marietta Energy Systems, Inc.
for the
U. S. Department of Energy
under Contract No. DE-AC05-84OR21400

Date Published: October 1992 


\section{Contents}

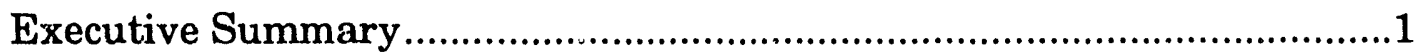

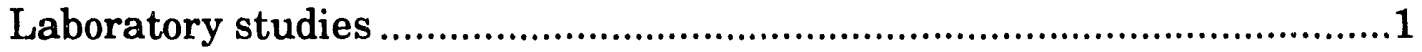

Design recommendations.....................................................................2

Prospective operations problems............................................................

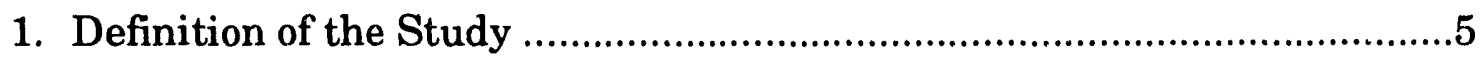

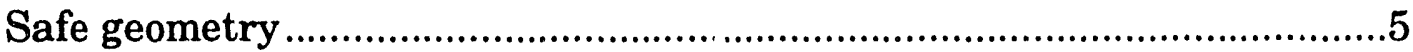

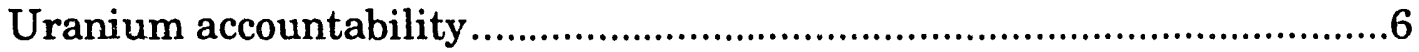

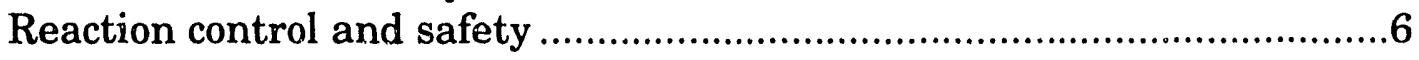

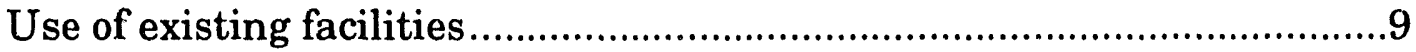

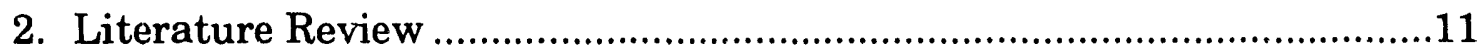

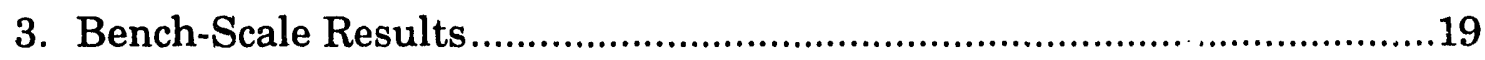

Preliminary manual pot dissolving tests.............................................19

Larger tests with computer controlled pump............................................20

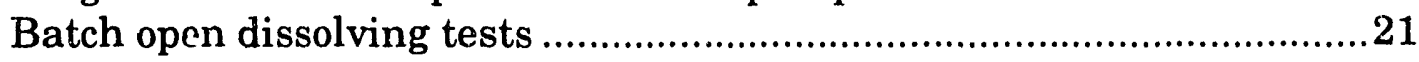

Large scale safe geometry cylindrical dissolver ......................................21

Experimental difficulties ..................................................................22

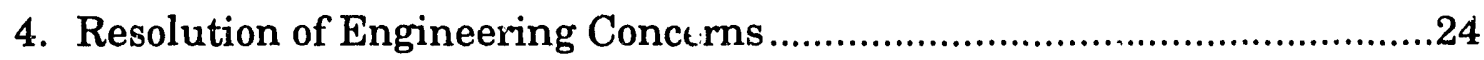

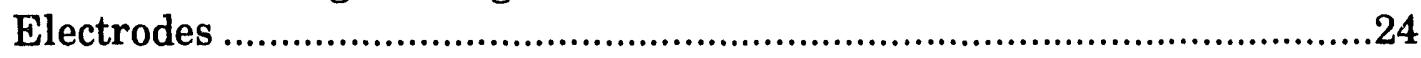

Computerized pump control .............................................................26

Feroxide and nitric acid needs ...............................................................

Industrial $\mathrm{NO}_{\mathrm{x}}$ measurement methods............................................27

System configuration and mechanics .................................................28

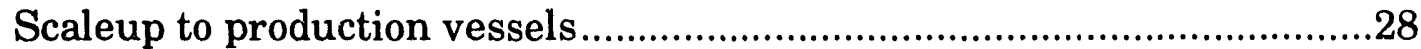

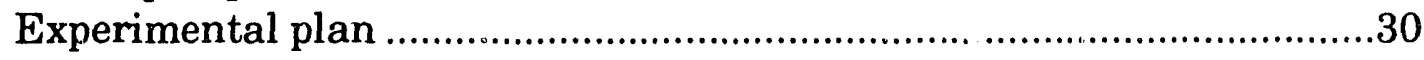

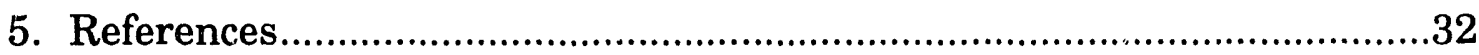

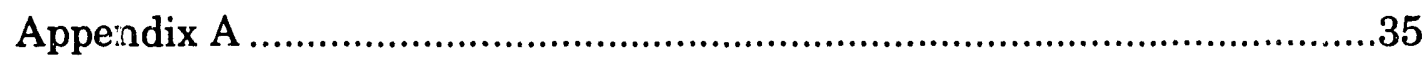

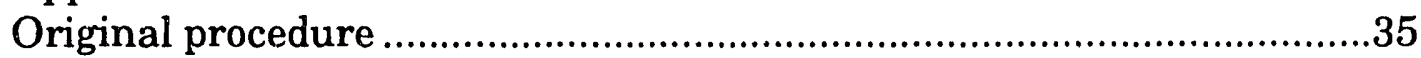

Manual addition peroxode, proposed procedure …….............................35

Continuous addition of peroxide, proposed procedure ...............................37

Automatic addition peroxide, proposed procedure....................................39 


\section{Executive Summary}

This study was designed to assess the feasibility of decreasing $\mathrm{NO}_{x}$ emissions from the current uranium alloy scrap tray dissolving facility. In the current process, uranium scrap is dissolved in boiling nitric acid in shallow stainless-steel trays. As scrap dissolves, more metal and more nitric acid are added to the tray by operating personnel. Safe geometry is assured by keeping liquid level at or below $5 \mathrm{~cm}$, the depth of a safe infinite slab. The accountability batch control system provides additional protection against criticality. The trays are steam coil heated. The process has operated satisfactorily, with few difficulties, for decades. Both uranium and uranium alloys are dissolved. Nitric acid is recovered from the vapors for reuse. Metal nitrates are sent to uranium recovery.

Brown $\mathrm{NO}_{\mathrm{x}}$ fumes evolved during dissolving have occasionally resulted in a visible plume from the trays. The fuming is most noticeable during startup and after addition of fresh acid to a tray. Present environmental regulations are expected to require control of brown $\mathrm{NO}_{\mathrm{x}}$ emissions. Because $\mathrm{NO}_{\mathrm{x}}$ is hazardous, fumes should be suppressed whenever the electric blower system is inoperable.

Because the tray dissolving process has worked well for decades, as much of the current capital equipment and operating procedures as possible were preserved. A detailed review of the literature, indicated the feasibility of slightly altering process chemistry to favor the production of $\mathrm{NO}_{2}$, which can be scrubbed and recycled as nitric acid. Methods for controlling the process to manage offgas product distribution and to minimize chemical reaction hazards were also considered.

\section{Laboratory Studies}

To determine whether it was possible to control process offgas composition by using hydrogen peroxide or oxygen gas to raise the redox potential of the reaction, a series of small bench reaction tests were performed. These tests started by simply dissolving a small amount of uranium in hot nitric acid, adding a sparge gas, and passing the offgas from dissolving through a distillation column. The gas was then sparged through a potassium hydroxide solution to produce nitrite so that it could be measured. Preliminary tests indicated that even the simple use of oxygen gas considerably decreased fumes from dissolving. 
Both the literature and previous experience indicated that hydrogen peroxide was useful in decreasing fumes. However, peroxide slows the rate and which uranium and its alloys dissolve. Excess peroxide causes the precipitation of uranium peroxide. At high concentrations, hydrogen peroxide can cause "runaway reactions" or react violently with nitric acid. To minimize the amount of hydrogen peroxide used, several control strategies which involved sensing redox with a redox electrode, determining an operating band, and controlling redox by small additions of peroxide, was developed. The peroxide metering system was readily controlled by a standard personal computer system equipped with data acquisition and control cards using a standard lab management software. The system satisfactorily operated a small pump to meter peroxide into the reaction in response to redox changes. The system was used with different bench-scale configurations and performed well.

To mimic the open tray dissolvers, a series of tests using a heated, open stainless-steel beaker were also performed. These tests indicated the need for more peroxide because evaporation increased the concentration of nitric acid in the beaker. Otherwise, the performance was similar to that of the closed vessel dissolving tests.

Because the literature survey indicated the possibility of continuous, safe geometry dissolving operations in a 4-inch diameter cylindrical dissolver, one was constructed and tested using a larger sample of depleted uranium. The dissolver and the process control system worked well and should be considered as a future alternative. A continuous system would minimize operator time and effort and, because it is fully contained, would minimize fume generation.

\section{Design Recommendations}

The goal is a continuous, safe-geometry dissolver with instrumented control of the dissolving process, including its offgas emissions. We propose to reach this goal by a series of simple steps which will decrease emissions and increase operator safety. Progress at each step will be evaluated and the operating procedure modified to enhance process management. The first step is installation of redox electrodes in a tray dissolving unit. This will involve modifying the cover of the tray dissolver to allow adding fittings to insert the electrode. The possibly of adding a horizontal mixer will be explored. Using an operating procedure modified to cover electrode management, peroxide will be added to keep the process redox measurement within the operating band. 
A single tray dissolver, converted for use as a prototype system has been arranged to permit evaluation of a number of control strategies. Automatic measurement of redox and control of tray temperature, liquid level, and peroxide addition rate will permit comparison of the effectiveness of several simple control strategies. These may eliminate the need for continuous redox monitoring. For example, by controlling tray temperature, it may be possible to stabilize the peroxide decomposition rate. Desired redox levels could then be maintained by addition of peroxide at a constant rate.

When the prototype is operating well using computer-assisted peroxide addition, other tray dissolvers can be similarly modified. Since the scrap dissolving operations are supervised by the same small group of operators, the prototype should serve as both an experimental and a training facility.

\section{Prospective Operations Problems}

Prototype tests are expected to resolve some fundamenial design and operating questions that are not readily answered by bench tests. Different materials and bridge electrolyte solutions for redox electrodes are available. Prototype operation will assess the utility of different materials (plastic electrodes) and of different electrolyte solutions and different locations and orientations. If conventional chloride electrolyte in a standard electrode body provides acceptable performance, then its lower cost and ease of replacement will favor use. If a nitrate-bridge electrolyte is needed, it can be used. Peroxide will be added at the same point as fresh nitric acid. If convective mixing is inadequate, a simple manifold to permit addition of the mixture at several, rather than one, point will be designed and tested. If mixing is still inadequate, magnetic or horizontal mixers will be assessed.

The selection and design of the peroxide supply system will have a major impact on process operations. In small bench tests, accumulation of bubbles in the flow lines caused problems because they displaced peroxide and reduced the peroxide delivery of the pump. The gas also made it difficult to prime the pump. Although the very small equipment used in bench tests contributes to these problems, any peroxide metering system needs to be designed to minimize this problem. Appropriate pump design measures include flooded suction and adequately sized tubing. However, simple gravity feed systems may provide better performance.

Although several types of equipment of monitoring $\mathrm{NO}_{\mathrm{x}}$ emissions are commercially available, they are often difficult to use; designed for measuring clean, conditioned, offgas; and of poor reliability. Prototype tests will include evaluation of a new type of $\mathrm{NO}_{\mathrm{x}}$ sensors developed for automobile emissions measurements as these may be more reliable or less expensive than 
conventional technology. However, it is expected that "best available technology" $\mathrm{NO}_{\mathrm{x}}$ moritoring will be installed as a part of the facility upgrade.

In summary, this bench investigation showed that manipulation of tray dissolver chemistry can sharply decrease offgas $\mathrm{NO}_{\mathrm{x}}$ fumes. The techniques used can be readily transferred from bench-scale tests to full scale operations. We recommend that each step be prototyped on a single modified dissolver tray and that, after supervision is satisfied with performance, other tray dissolvers can be modified. Although we found a fully automated system preferable, it is important to remember that most of the environmental benefit comes from the simple use of a redox-based control system. 


\section{Definition of the Study}

This effort is directed toward optimizing the uranium alloy tray dissolving process to decrease the visibility of $\mathrm{NO}_{\mathrm{x}}$ stack emissions. Batchcontrolled uranium and uranium alloy scrap are cut into small pieces, weighed, and batched. Portions of the scrap from a given batch are heated in $15 \%$ nitric acid in $60 \times 60 \times 5 \mathrm{~cm}$ safe-geometry stainless-steel pans. Fresh acid and metal pieces are periodically added and overflow uranium nitrate solution is collected, filtered, and sent to uranium recovery. The dissolving trays are in hoods and have their own exhaust system which includes a wet scrubbing tower. The scrubbing liquid starts as 0.1 to $5 \%$ peroxide in water. When the nitric acid concentration reaches $10 \%$, the scrubber liquid is recycled. System design was originally optimized to provide ease of operation, uranium accountability, and operator safety. The process has operated as designed for many years with few difficulties. However, changing environmental and materials reuse requirements are making emissions minimization attractive.

Proposed process modifications are expected to conform to current administrative constraints, including maximizing the use of existing facilities and following uranium accountability and safety requirements. Good engineering practice indicates preserving the safe-geometry and operator safety features incorporated in the current process design. Individual pans need to be monitored and controlled so that a runaway reaction does not occur. A batch control system is used for accountability. The limitations imposed by these and other historical constraints and their effect on current and proposed processes are discussed below.

\section{Safe Geometry}

The first, and foremost, safety strategy used in the uranium dissolving process is safe geometry. In the current dissolving process, the stainless steel pans provide a liquid level of approximately $5 \mathrm{~cm}$, the depth of a safe infinitesize slab. This provides protection against a critical accident even if a failure in analysis or batch control occurs. The liquid level is maintained by manual operator control of nitric acid additions and product removals and by a liquid level overflow. Open vents have also been placed slightly above the liquid overflow level to further ensure that the tray liquid level remains safe. 
A safe-geometry dissolver could also be constructed as a nominally 4 inch diameter cylinder. This design, as reported by other investigators, may enhance mixing and improve redox electrode reliability.

\section{Uranium Accountability}

In the current process, uranium accountability is maintained by batch analysis coupled with a batch control system. A batch of scrap is cut, weighed, and portioned. Analysis of a represeatative sample is used to control the amount of uranium per batch. The system provides both accountability and a second method for preventing a critical accident. This could be very valuable if the depth of liquid in the dissolver increased due to, for example, manual addition of an excess of nitric acid together with blockage of overflow tubes and vents.

The batching - accountability system for uranium management presents, at most, a minor handling nuisance. However, the benefits, in terms of safety and a simple, effective method for meeting mandatory administrative requirements, are significant. Any new process can be adjusted to accommodate the batch accountability system.

\section{Reaction Control and Safety}

Although there have been few historic problems in operating the current tray dissolver system, other batch dissolving systems have experienced some process difficulties. Any successful design needs to address the major process chemical reaction problerns, runaway reaction and violent reaction between peroxide and nitric acid.

Runaway reaction. The dissolution of uranium in nitric acid is an exothermic, or heat generating, reaction. The heat generated by the reaction can increase the rate at which the reaction occurs. This has been managed by a combination of good operator control and good operating practice. For example, the reaction is generally started cold and gradually increased in temperature until a satisfactory dissolution rate is reached. The operator controls the rate of reaction by managing the rate of nitric acid addition and the process operating temperature.

Peroxide handling and metering. The Y-12 Plant has had a long history of peroxide use in uranium processing. Some peroxide handling problems, partially due to the high concentrations of peroxide used, have also occurred. Undiluted peroxide addition to a boiling scrap dissolving pot was standard practice. This caused some handling problems, ir.cluding uranium oxide precipitation and uneven reaction. 
Hatcher and MacLaughlan (1938) studied the decomposition of mixtures of nitric acid and hydrogen peroxide. They found that, at high concentrations of both reagents, mixtures of nitric acid and hydrogen peroxide react violently. Figure 1.1, from their data, shows the limits of violeni, reaction of nitric acid and hydrogen peroxide. Below curve $B$ the mixtures were termed unreactive at room temperature. At higher tømperatures, reaction rates are likely to increase. However, no problems were encountered in our lab-scale experiments using $30 \%$ peroxide and $30 \%$ nitric acid up to the boiling point. Limiting peroxide to $30 \%$ and limiting peroxide addition rate during dissolver startup, coupled with controlling the

ORNL-DWG 92M-13304

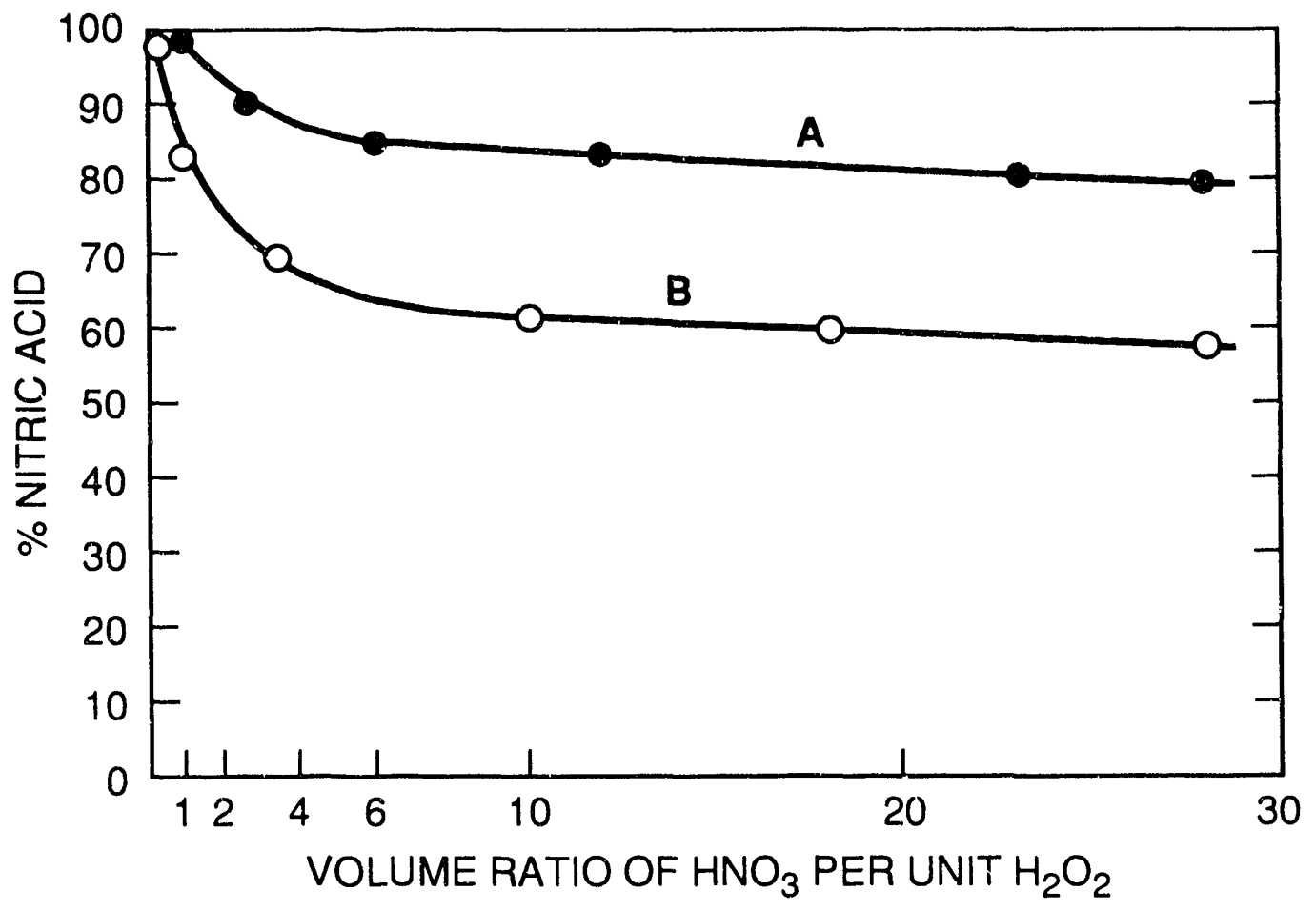

Fig. 1.1. Reacting volumes of nitric acid and hydrogen peroxide; showing volumes of aqueous nitric acid of various concentrations added to one volume of $62.9 \%$ hydrogen peroxide at 21 to $23^{\circ}$ C. Curves: $A$, lower limit of instantaneous reaction; B, lower limit of any noticeable reaction. Source: Hatcher and MacLaughlan (1938).

addition so that only small aliquots of peroxide are required to eliminate $\mathrm{NO}_{\mathrm{x}}$ fumes, and keeping the concentration of nitric to $50 \%$ or less, should sharply decrease or minimize uneven reaction. This can be accomplished by shortening the periods between peroxide addition and decreasing the size of peroxide aliquots. 
Schumb, Satterfield, and Wentworth (1955), in their ACS monograph on hydrogen peroxide, provided limits for the safe use of peroxide. They indicated that it was difficult to detonate even very high concentrations of liquid peroxide. However, they indicated that the vapor, above $26 \% \mathrm{v} / \mathrm{v}$ could be detonated. The corresponding equilibrium aqueous concentrations are shown in Fig. 1.2. Detonatable vapors are not formed at aqueous concentrations below approximately $75 \%$ peroxide at one atmosphere.

ORNL DWG 92M- 13839

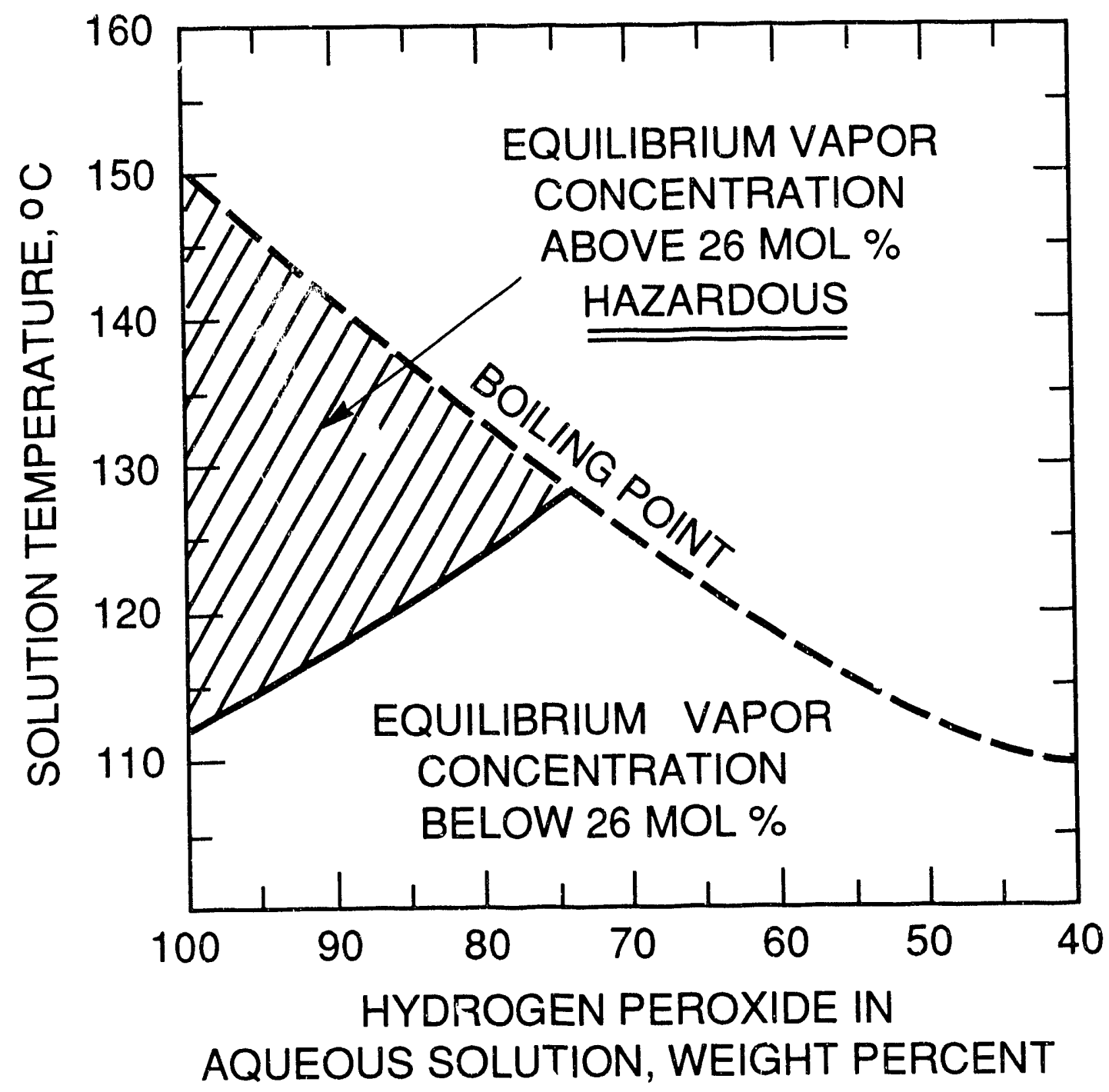

Fig. 1.2. Combination of temperature and liquid composition producing equilibrium vapors in explosive region at 1 atmosphere. Source: Schumb, Satterfield, and Wentworth 1955. 
Although there is little or no organic material present in the tray dissolvers, organic materials are reactive with peroxide and nitric acid. Detonations have not been observed in mixtures containing less than approximately $27 \%$ hydrogen peroxide. All of these studies indicate that keeping the tray dissolvers free of organic materials and keeping the concentrations of both peroxide and nitric acid below $30 \%$ will preclude the possibility of violent reaction or detonation.

$\mathrm{NO}_{\mathrm{x}}$ hazard. The Clean Air Act mandates decreased $\mathrm{NO}_{x}$ emissions from industrial and combustion processes. This is a major change because $\mathrm{NO}_{\mathrm{x}}$ emissions from small industrial processes are presently unregulated. The tray dissolving process is double-contained in a hood and with an upper cover to protect the operator from $\mathrm{NO}_{\mathrm{x}}$ exposure above the OSHA limit. The operator exposure limits are substantially more restrictive than the emissions control limits. Additionally, the operator should be better protected when hood ventilation fails. This is important because the scrap dissolving pans are steam heated and the steam heat will not be affected by an electrical outage.

By moving primary control of $\mathrm{NO}_{\mathrm{x}}$ emission from the scrubber to the chemical process itself, generation can be more effectively controlled during periods of power failure, building evacuations, etc. This will also limit the amount of non-nitric acid $\mathrm{NO}_{\mathrm{x}}$ enter the scrubbing tower and should maximize the nitric acid recovery.

\section{Use of Existing Facilities}

Existing facilities provide some major advantages: they are in place, immediately usable, and paid for. The facilities include safe-geometry tray dissolvers, hoods, a ventilation system, and a scrubber with storage tanks. Scrubber efficiency is unlikely to be substantially increased without a major capital addition. The tray dissolvers are a variable, readily controlled unit process which tolerate a variety of operating conditions.

Our first questions concerned process management needs. Control of $\mathrm{NO}_{\mathrm{x}}$ emissions, which are likely to be regulated in the next two years, was a major priority. Recovery of $\mathrm{NO}_{\mathrm{x}}$ as nitric acid would help to meet Resource Conservation and Recovery Act (RCRA) requirements. Minimization of brown NO ernissions, a controlling factor in scrubber operation, would be likely to permit recovery of more concentrated nitric acid which could be used in a wider range of plant operations. Priority was also given to maximizing operator convenience by automating process control and logging. Approaches amenable to safe-geometry and continuous processing were also emphasized. 
We surveyed available uranium dissolution literature to obtain conceptual ideas for $\mathrm{NO}_{\mathrm{x}}$ reduction and chemical modeling data. We also contacted the manufacturers of small process and control equipment. We made a preliminary selection and designed and performed a series of bench experiments to help optimize the proposed equipment and chemical process operations to meet Y-12 Plant needs. This report details the effort and provides recommendations for process tests of the proposed equipment. 


\section{Literature Review}

We surveyed the professional literature for reports covering the dissolution of uranium and its alloys in nitric acid solutions with and without the addition of peroxide. We were particularly interested in methods or processes for decreasing the emissions, handling, or time required for metal dissolution and sor methods which and the promise of increasing operator safety or convenience. We were also interested in nitric acid - water peroxide systems. We felt that modification of the dissolving process chemistry, as opposed to the increasing the size and capacity of the existing scrubber, offered the best chance of success.

Most of the literature discussed below describes continuous and semibatch dissolvers. These have historically been used in processes which handled native and low-enrichment uranium and uranium alloys. However, the chemical reactions on which design of both continuous and batch tray dissolvers is the same for highly enriched uranium except for safe-geometry constraints. Over the years, a variety of methods, including acid dissolving, base dissolving, and electrochemical methods, have been used in both low and high enrichment systems. Nitric acid dissolution continues to predominate because it produces uranyl nitrates which can be readily purified by solvent extraction.

Miles' pioneering research into processes for the fumeless dissolving of ur anium metal in the presence of oxygen is perhaps the most relevant to this study (done in 1949, declassified and published in 1955, 1956 and Martin and Miles 1958). His process was adapted from British patents 634189,634578 , and 634579 filed by Johnson and Sons, Ltd., for the dissolution of silver and other heavy metals. The dissolving apparatus was a short jacketed column filled with the uranium metal spheres to be dissolved. Nituic acid was passed down over the uranium spheres and uranyl nitrate was withdrawn at the bottom of the process column. A second packed bed column was placed above the metal dissolution column to oxidize and strip process offgas as well as to preheat acid going into the metal dissolution column. A batch process pot. dissolution process in which the uranium was totally immersed could be substituted for the metal dissolution column with limited effect on offgas removal. However, efficient operation of the pot dissolution process required direct introduction of concentrated nitric acid into the pot, rather than the scrubber. In both cases, colored $\mathrm{NO}_{\mathrm{x}}$ fumes normally formed during the dissolution process were reacted with oxygen and recycled as process makeup acid. 
The work of Blick, Clucas, McDonogh, and Wocds on reducing emissions from nitric acid pickling operations is closely analogous (1992). These investigators explored the addition of peroxide to scrubbing towers and to nitric acid - hydrofluoric acid baths used for pickling stainless steel. The found that continuous direct addition of peroxide to a recirculation strean: maintained $\mathrm{NO}_{\mathrm{x}}$ emissions between 10 and $100 \mathrm{ppm}$. Uncontrolled streams were above $500 \mathrm{ppm}$. Scrubber systems with peroxide addition could be used to maintain a constant $\mathrm{NO}_{\mathrm{x}}$ output of $30 \mathrm{ppm}$. Both peroxide addition systems operated at full scale without process control other than peroxid metering.

Blick and coworkers drew heavily on the research of Kemperman (1976). Kemperman claimed processes in which metal dissolution in nitric acid was facilitated by the addition of 1 to 20 grams per liter of hydrogen peroxide. Temperatures from 10 to $70^{\circ} \mathrm{C}$ and a variety of dissolving and cleaning solution compositions were reported. Peroxide stabilizers, including ethylene glycol, did not increase the evolution of nitric acid fumes from dissolving baths. The preferred mole ratio of peroxide to nitric acid was 0.273. The bulk of the solutions surveyed were "bright dips" which are used to clean copper by dissolving the surface metal and oxide layers.

Dalin (1988) described a system for using redox measurements for controlling available peroxide concentrations in a nitric acid system used to pickle stainless steel. Although the process configuration was somewhat different than the one which we are proposing, the control technology is analogous. Using this peroxide addition system, it was possible to reduce brown $\mathrm{NO}_{\mathrm{x}}$ emissions below $20 \%$ of the uncontrolled value. This permitted the facility to meet its discharge limits.

To minimize the production of nitrogen oxides, Hofmann, Holtz, and Wagner (1979) designed a process for the dissolution of irradiated $\mathrm{UO}_{2}$ reactor fuel elements. This process also facilitates the recovery of $\mathrm{Kr}$ and $\mathrm{Xe}$ gases. Direct addition of $\mathrm{H}_{2} \mathrm{O}_{2}$ to the dissolver is used to replace the oxidizing provided by the nitric acid conventionally required. The $\mathrm{H}_{2} \mathrm{O}_{2}$ addition is managed so that the process offgas consists predominantly of $\mathrm{O}_{2}$ with approximately $10 \%$ noble gases.

Hufner (1982) evaluated a number of alternatives for the processing of dissolver offgas from fuel element reprocessing. He found the volume of dissolver offgas per weight of heavy metal dissolved to be a major factor in the selection of an appropriate process design. He found concurrent and countercurrent $\mathrm{NO}_{x}$ stripping with extremely cold $\left(-55^{\circ} \mathrm{C}\right) 90 \%$ nitric acid to be an effective alternative to the more conventional countercurrent mild caustic stripping. The cold acid stripping was also effective in removing 
iodine from the dissolver offgas. Hufner presents pilot results on tests of a number of process alternatives.

Wymer and Foster reviewed dissolution of spent reactor fuel elements in both acid and base (Wymer and Foster 1956, Foster and coworkers 1956). They were interested in understanding the chemical kinetics of uranium alloy dissolving reactions in order to improve the design of continuous dissolvers for reprocessing irradiated reactor fuel elem ints. As might be expected, they noted that the dissolution rate of uranium metal increases with increasing nitric acid concentration and decreases with increasing uranyl nitrate concentration. They noted that a number of chemical additives, including $\mathrm{HF}, \mathrm{CaF}_{2}, \mathrm{Na}_{2} \mathrm{SO}_{4}, \mathrm{H}_{3} \mathrm{PO}_{4}$, and ammonium silico fluoride, had been used to accelerate the rate of uranium metal dissolution, while small amounts, typically $0.5 \mathrm{mM}, \mathrm{Hg}\left(\mathrm{NO}_{3}\right)_{2}$ were used to accelerate the rate of aluminum-uranium alloy dissolution.

As their studies continued, Wymer and Foster found that a number of process geometries, including pot, upflow column, downflow trickle, and slab dissolvers could all be used to dissolve uranium metal. To maintain safe geometry, the size of the equipment would decrease with increasing uranium enrichment. In most configurations, nitric acid could be recovered by oxidation of offgas nitrogen oxide, followed by water absorption.

Subsequently, Wymer and Blanco (1957) studied the dissolution 5-15\% cast and extruded uranium-aluminum alloys in nitric acid using a $5 \mathrm{mM}$ $\mathrm{Hg}\left(\mathrm{NO}_{3}\right)_{2}$ catalyst. They found that the catalyst increased the reaction rate by an order of magnitude. Although the overall rate of dissolution was a function of the history of the metal sample, the maximum rate of dissolution for most alloys tested occurred between 6 and $8 \mathrm{MHNO}_{3}$.

In these studies, Wymer and coworkers showed that a wide variety of low enrichment fuel alloy types and shapes could be readily dissolved in safegeometry vessels using nitric acid. Although the kinetic equations are useful in estimating dissolver performance, they are predominantly empirical fits to data observed under the specific conditions studied. At the time of these studies, management of non-radioactive emissions was not a major concern.

The research of Foster and Nurmi (1952) on the dissolution of cast and extruded uranium alloy slugs provided a base comparison of nitric acid and caustic dissolving processes. The nitric acid dissolution studies involved the use of extremely low nitric acid and extremely high mercuric nitrate concentrations. These studies showed that, as nitric acid concentration was decreased, the optimum concentration of mercuric nitrate catalyst increased. Process sensitivity to addition of oxygen to the offgases was also studied. These runs indicated the possibility of using oxygen to treat offgases. 
Bresee, Foster, and Nurmi investigated continuous dissolution of uranium-aluminum fuels by nitric acid in a trickle-type column dissolver $(1956,1959)$. Cast or extruded slugs were added to the top of the 5.5 inch ID by 10 foot, 8 inch tall column. Nitric acid concentrations ranging between 4 and $8 \mathrm{M}$ with $0.005 \mathrm{M} \mathrm{Hg}\left(\mathrm{NO}_{3}\right)_{2}$ catalyst were pumped into the bed at flow rates ranging between 0.1 and 7 liters $/ \mathrm{min}$. Sufficient diversity in results among runs on extruded slugs was observed to indicate that the dissolution rate was proportional to an exponential of the acid feed rate; however, the exponent varied with acid concentration. The ratio of acid consumed to metal dissolved indicated the possibility of different concurrent reaction mechanisms.

Gens and Clark (1959) detailed the Perflex Process, which employed a mixture of $\mathrm{HF}$ and $\mathrm{H}_{2} \mathrm{O}_{2}$ for dissolving predominantly zirconium alloys containing $7 \%$ uranium and $2 \%$ or less tin. The final dissolver solution was to be mixed with acid aluminum nitrate raffinate to form a solution from which the uranium could be recovered by solvent extraction. Use of peroxide with $\mathrm{HF}$ increased the solubility of uranium, together with the rate of dissolution of all of the alloy metals. The investigators determined corrosion rates of a number of materials in an effort to find a satisfactory material of construction. Although Hastelloy $\mathrm{C}$ gave the best results, 0.08 mils per cycle, corrosion rates were somewhat variable. Corrosion rates for Haynes 25, 90 $\mathrm{Ag}-10 \mathrm{Cu}$, INOR 8, and Monel were too high for effective use. In bench work, teflon was the preferred material of construction.

Zhukovskii and coworkers (1987) modeled the effects of temperature, particle size, and nitric acid concentration on the dissolution of uranium dioxide particles in nitric acid. Particle diameters modeled ranged up to 1.5 $\mathrm{mm}$, while nitric acid concentrations ranged between 6 and $12 M$. Process temperatures fell above $60^{\circ} \mathrm{C}$. Models were based directly on experimental data and their validity was confirmed by their accurate prediction of fresh experimental data. A series of equations covering the dissolution of uranium dioxide powders was presented.

Swanson and coworkers (1985) investigated dissolution kinetics of uranium reactor fuel in nitric acid. The work was performed to provide a basis for design of a large-scale dissolver for reactor fuel. Dissolution was found to be proportional to the square of nitric acid concentration, or to the 2.6 power of bulk nitrate ion activity, in the experimental range of 3 to $8 M$. This indicates that the uranium dissolution rate is proportional to the first power of nitrate (or hydrogen) ion activity, and that the primary contribution of uranyl nitrate in increasing the rate of reaction is due to its contribution of nitrate ions. 
Increases in temperature below the boiling range of nitric acid increase the rate of uranium dissolution. A maximum rate occurs a few degrees below the boiling point of nitric acid. This appears to result from an increase in reaction rate due to the presence of a small amount of nitrous acid in the dissolver solution. This was confirmed by addition of hydrazine to a working dissolver; after hydrazine addition destroyed nitrous acid, the dissolution reaction stopped. Swanson and coworkers did not determine whether the function of nitrous acid was catalytic.

Correlation between reaction rate and exposed uranium surface was, as expecteci, found. Although these investigators found an expected correlation between surface roughness and reaction rate, accounting for some of the increase in reaction rate during dissolution, the effect was limited by the large uranium grain sizes in the fuel elements. This resulted in a threefold increase in surface which occurred after a dissolution depth of approximately 50 microns.

In spite of the chemical complexities of the dissolving and acid recycle systems, it appeared possible to control the dissolution of aluminum uranium alloys by 1 ) feeding slugs at a uniform rate while allowing the packed bed to seek its own height; 2) adjusting acid feed rate to produce the desired product concentration; and 3) setting the final acid concentration in the solution based on the assumption of 3.85 moles of acid consumed per mole of metal fed. Dissolution rate during the process could be calculated as a function of increases in condenser water heat using the assumption of 190 $\mathrm{kcal} / \mathrm{gram}$ mole aluminum dissolved.

Caracciolo and Owen (1970) described methods for the electrolytic dissolution of power reactor fuel elements in nitric acid. Electrolysis was investigated because of the tough oxide films which make fuel element metal surfaces resistant to acid dissolution. The experimenters report a series of tests on real and simulated reactor fuels using a $5000 \mathrm{~A}, 25 \mathrm{~V}$ pilot scale dissolving unit outfitted with niobium basket and platinum sheet electrodes. The basket also supports fuel element pieces. Using a range of nitric acid concentrations and operating parameters, the assembly successfully dissolved most current fuel element configurations. Little or no hydrogen offgas evolution was noted. The pilot was essentially a smaller section of a proposed larger installation to minimize design difficulties during scaleup. Corrosion of the electrodes was in the 0.5 to 2.5 mil per year range, indicating that equipment life should be satisfactory. Methods for dealing with the sludges produced during dissolution of some types of reactor elements were also described.

Wymer and coworkers also considered the dissolution kinetics of uranium-aluminum alloys in 3 to $8 M$ caustic (Wymer and Blanco 1957, 
Wymer and coworkers 1959). The reaction involves dissolving the aluminum to form sodium aluminate and precipitating the uranium as uranium (IV) oxide:

$$
\mathrm{Al}+\mathrm{NaOH}+\mathrm{H}_{2} \mathrm{O} \rightarrow \mathrm{NaAlO}_{2}+1.5 \mathrm{H}_{2} .
$$

The uranium oxide particles, typically $100 \mathrm{~nm}$, are recovered by gravimetric methods such as centrifugation. Separated uranium oxide was dissolved in $6 M$ nitric acid as a solvent extraction feedstock. In the presence of excess caustic, sodium aluminate solutions are stable at room temperature for extended periods. If an excess of caustic is not present, the sodium aluminate is gradually converted to solid aluminum oxide trihydrate and caustic. The reaction appeared to be first order with respect to caustic concentration and the rate did not appear to be substantially affected by the metallurgical history of the sample. The rate of dissolution was not appreciably affected when hydrogen peroxide up to ten times that produced by fission products was added. Sodium carbonate similarly had no appreciable effect on the rate of reaction. When dissolution was affected at near-boiling temperatures, catalytic amounts $\left(6 \times 10^{-4} M\right)$ of $\mathrm{NaI}$ were used to assure uranium oxide precipitation. The uranium oxide precipitate contained, at most, very low levels of impurities. Several methods for dealing with the hydrogen gas liberated in the initial aluminum dissolution were described.

Schortmann and DeSesa evaluated the effect of carbonate and bicarbonate ions on the kinetics of $\mathrm{UO}_{2}$ dissolution (1958). They used a finely divided $\mathrm{UO}_{2}$ powder and performed their experimental work at $>80^{\circ} \mathrm{C}$ with an oxygen overpressure of 0.5 to $13.5 \mathrm{~atm}$. The reaction was found to be first order with respect to particle surface area. Temperature dependence followed the Arrhenius rule and the reaction's activation energy was found to be independent of oxygen overpressure. In the ranges between 0 and $0.5 M$ carbonate and 0 to $0.5 \mathrm{M}$ bicarbonate, reaction rate increased as a function of increasing carbonate and bicarbonate concentration. It was, however, necessary to maintain $\mathrm{pH}$ above 9 to prevent inhibition of the reaction. The authors postulated that the reaction involved the oxidation of uranium (IV) to uranium (VI) followed by the formation of a soluble uranyl dicarbonate complex.

Baker and Newton (1961) investigated the kinetics of reaction between $\mathrm{U}^{+4}$ and $\mathrm{H}_{2} \mathrm{O}_{2}$ in $\mathrm{HCl}$ solutions. They found that consumption of peroxide was essentially constant across the range of conditions tested. The proposed second-order rate dependence on $\mathrm{H}_{2} \mathrm{O}_{2}$ concentration was found to poorly represent experimentally measured kinetics. Rates were substantially increased by the presence of small amounts of $\mathrm{Ag}^{+1}, \mathrm{Mn}^{+2}$, and $\mathrm{Fe}^{+2}$ and somewhat decreased by larger amounts of $\mathrm{Cu}^{+2}$ and $\mathrm{Co}^{+2}$. Increasing the concentration of $\mathrm{HCl}$ in the dissolving solution did, however. increase the rate 
of reaction. No correlation between reaction rate and ionic strength, dissolving vessel glass surface area, or light intensity was apparent. The chain carriers were found to be $\mathrm{U}(\mathrm{V})$ and $\mathrm{HO}_{2}$. The authors felt that their data indicated a that the dissolution reaction had at least two steps.

Eligwe, Torma, and De Vries (1982) investigated the use of peroxide to facilitate sulfuric acid leaching of uranium from a New Mexico ore. Their results indicated mass transfer to be controlling in uranium leaching. They also found the reaction sequence to be a multistep process with a very low activation energy. Overall, $\mathrm{H}_{2} \mathrm{O}_{2}$ improved yield by $5 \%$ and increased the uranium extraction rate significantly between 30 and $50^{\circ} \mathrm{C}$ in a stirred leaching vessel. At temperatures above $50^{\circ} \mathrm{C}$, significant peroxide decomposition occurred. For near-ambient temperatures, peroxide provided a significant improvement over sodium chlorate, a more conventional additive. It also provides a method of oxidizing materials, such as iron, which coleach with uranium.

Eligwe and Torma (1986) reviewed the status of methods for leaching uranium ores with $\mathrm{H}_{2} \mathrm{O}_{2}$ in alkaline and acid solutions. They indicated that peroxide usage, although confined largely to solution mining operations, was likely to be practical for use in above-ground leaching operations. They indicated that the presence of a small amount of iron in leach solutions increased the reaction rate, although large amounts of iron or iron sulfide in ores could consume a substantial amount of peroxide. The precipitation of uranium oxide in the presence of excess peroxide in alkaline solutions appeared to be a major process control problem. The authors indicated that, because wistor and oxygen are its most common breakdown products, hydrogen peroxide was among the most environmentally benign leaching solutions.

Carson, Norris, and Schellinger (1982) investigated the leaching of uranium from ore in the presence of $\mathrm{H}_{2} \mathrm{O}_{2}$ as a part of research on solution mining of low-yield uranium ores. In this process, ore 60 to $600 \mathrm{~m}$ below ground level was leached with a sodium or ammonium carbonate solution to selectively yield uranyl carbonate. The porous and finely granular structure of the ore permitted easy passage of leach solutions and encourages good mass transfer. High uranium leachate was collected in wells for further processing. Uraniurn recovery in the presence of $\mathrm{H}_{2} \mathrm{O}_{2}$ equivalent to $20 \mathrm{mg} / \mathrm{l}$ of dissolved oxygen was greatest at $\mathrm{pH}$ values above 7.5 or below 5 . However, formation of insoluble uranium oxides at low $\mathrm{pH}$ tends to favor use of basic leach solutions. Uranium leaching was directly proportional to consumption of $\mathrm{H}_{2} \mathrm{O}_{2}$; rate increased with the amount of alkaline carbonate buffer used in the leaching solution. In other tests, the effect of oxidant on the amount of uranium leached was shown to decline after removal of the bulk of the uranium. 
The bulk of the research summarized above has been directed toward the dissolution of previously irradiated uranium alloys, such as spent reactor fuels. Although release of daughter products was a major concern, release of nitrous oxide was not. The scrap in the present study is unirradiated scrap uranium and uranium alloys. Current legislation and DOE administrative mandates are placing increasing emphasis on environmental and safety concerns. Minimizing $\mathrm{NO}_{x}$ emissions, including those emissions which could threaten operator safety, has a high priority.

Based on literature reports, it is clear that effective uranium dissolution can be accomplished concurrently with minimization of $\mathrm{NO}_{x}$ fumes. The penalty reported in the literature is largely one of decreased rate of reaction. However, it appears that this can be minimized with modern process control technology.

Another intriguing possibility is the use of caustic dissolıtion of uranium-aluminum alloys. This would substantially reduce the generation of $\mathrm{NO}_{\mathrm{x}}$. This has the advantage of decreasing both emissions and the overall use of nitric acid but increases waste generation. 


\section{Bench-Scale Results}

From the literature survey, hydrogen peroxide appeared likely to provide effective control of $\mathrm{NO}_{\mathrm{x}}$ evolution by a nitric acid system. However, no comparisons of the effectiveness of fumeless dissolving technologies, like those pioneered by Miles, were available. Additionally, modern instrument logging and setpoint control techniques appeared likely to provide a method of optimizing peroxide addition using redox control. Nitric acid dissolving creates a harsh process environment and could substantially shorten equipment lifetimes. A short series of experiments to see which technology was likely to be most effective and to explore the problems associated with the use of these technologies for enriched scrap dissolving were performed.

\section{Preliminary Manual Pot Dissolving Tests (15 g)}

A bench scale uranium dissolver was designed to test novel process strategies. The small version of Miles' pot dissolver was made using conventionally available laboratory glassware. The size was scaled to a level which minimized waste generation while permitting comparison testing of a variety of commercial electrode and pumping control systems. The apparatus was constructed to permit direct comparison of air, oxygen, and peroxide for nitrous oxide control.

Experimental procedurt Small square plates of uranium were placed in a jacketed spherical reaction flask (Lab Glass ML-1284-702) with a four neck cover (Lab Glass ML-1286). Nitric acid, peroxide, and a controlled flow of oxygen or air were added through the necks. A rotometer was used to measure gas addition. The reaction temperature was maintained at about $85^{\circ} \mathrm{C}$ using a Lauda recirculating bath. Offgas was passed through a $15 \mathrm{~mm}$ diameter by $500 \mathrm{~mm}$ distilling column packed with glass Raschig rings and a reflux condenser. It was then sparged into a measured amount of $10 \% \mathrm{KOH}$. The resultant nitrite was measured directly by absorbance at $377 \mathrm{~nm}$ using the method developed by Altshuller and Wartburg (1960).

Concurrent minimization of $\mathrm{NO}_{\mathrm{x}}$ fumes and hydrogen peroxide addition was selected as the best control strategy. It appeared that oxidation-reduction potential, or redox, of the dissolving solution could be used for process control. An Ingold redox electrode was provided in the reaction vessel to monitor the reaction. Brown fumes were observed above 900 or $1000 \mathrm{mV}$, depending on the electrode used. (The redox potential at which this reaction is favored will change with changes in concentration, 
electrode, temperature, and other operating conditions.) A redox potential taken below $600 \mathrm{mV}$ by peroxide addition precipitated uranium as $\mathrm{UO}_{4}$. For the electrode we were using, a redox potential of around $875 \mathrm{mV}$ appeared to provide adequate control of $\mathrm{NO}_{x}$ fumes without uranium precipitation. The results of this series of experiments are shown in Table 3.1.

Table 3.1. Preliminary pot dissolving tests with Redox electrode ( 15 g U)

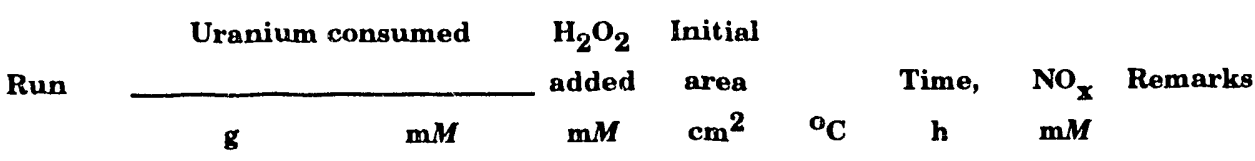

$\begin{array}{llrrrrrrl}\text { A } & 19.4 & 81 & 0 & 5.3 & 85 & 9.5 & 4.9 & \text { Air- no peroxide } \\ \text { B } & 15.0 & 63 & 176 & 4.7 & 85 & 11.7 & 0.34 & \text { Air- } 20 \mathrm{ml} 30 \% \mathrm{H}_{2} \mathrm{O}_{2} \\ \text { C } & 19.3 & 81 & 0 & 5.3 & 85 & 12.7 & 1.46 & \mathrm{O}_{2} \text { - no peroxide } \\ \text { D } & 15.3 & 65 & 352 & 4.8 & 85 & * & * & 7 M \mathrm{HNO}_{3} \text { - Redox control } \\ \text { E } & 12.7 & 53 & 582 & 4.5 & 90 & 7.1 & 0.84 & 12 M \mathrm{HNO}_{3} \text { - Redox control }\end{array}$

* Collection equipment failure; visual observation showed no fumes.

Air. Of the process strategies attempted, air is the closest to current practice. Although the performance should be a slight improvement over that of tray dissolvers due to the additional contact provided by the packed tower and the increased gas flow, runs made using air provide a baseline for performance comparison. Air + peroxide. All levels of peroxide tested in combination with air as a sparge gas provided a substantial increase in NO oxidation. Evolution of $\mathrm{NO}_{x}$ was reduced by over an order of magnitude. Oxygen. Atmospheric pressure oxygen reduced $\mathrm{NO}_{\mathrm{x}}$ evolution by a factor of three in comparison to the air-only control.

\section{Larger Tests with Computer Controlled Pump ( $>50$ g)}

A 96 hour batch dissolving test using redox potentials below $850 \mathrm{mV}$ to trigger addition of peroxide by a constant speed syringe pump was then performed. No nitric acid was removed during the test. In spite of early peroxide addition due to bubble formation in the syringe, $\mathrm{NO}_{\mathrm{x}}$ production remained low. The test indicated that simple limit controls on peroxide addition were effective in controlling $\mathrm{NO}_{x}$. Manual peroxide addition was needed for large peroxide additions at the beginning of a run. 


\section{Batch Open Dissolving Tests}

Open-beaker dissolving tests addressed the applicability of redoxcontrolled $\mathrm{H}_{2} \mathrm{O}_{2}$ addition to control fume development in an open nitric acid dissolution vessel similar to an open tray dissolver. The tests were performed at 90 to $95^{\circ} \mathrm{C}$ in a $400 \mathrm{ml}$ stainless steel beaker. The beaker was heated using a thermostated hot plate. During the experiments, 15 to $50 \mathrm{~g}$ uranium were dissolved in $100 \mathrm{ml}$ of $8 \mathrm{~N}$ nitric acid. This is approximately $1 / 100$ of tray dissolver scale. The open beaker arrangement did not permit collection and measurement of $\mathrm{NO}_{x}$.

During these tests, the liquid evaporated to about one-half its volume in about $4 \mathrm{~h}$. Since a constant amount of nitric acid was consumed by dissolution, the free nitric acid concentration increased. As a result, a larger amount of $\mathrm{H}_{2} \mathrm{O}_{2}$ was consumed by decomposition and more frequent additions of $\mathrm{H}_{2} \mathrm{O}_{2}$ were required to keep the redox potential in the control range. Addition of water or fresh acid to reestablish the original acid concentration decreased the necessary rate of peroxide addition. These batch tests demonstraied the feasibility of maintaining the acid concentration in a tray by varying the temperature and air flow rate to control evaporation. The rate of uranium dissolution was somewhat greater than that of batch pot dissolving tests because of the increased acid concentration. This effect was verified by running a blank test with only nitric acid (no uranium) in the beaker.

\section{Large Scale Safe Geometry Cylindrical Dissolver}

Larger scale tests were performed using a closed one liter vessel fitted with a bottom drain. The configuration and instrumentation were those used in the small pot dissolver tests except a standard heating mantle was used to heat the vessel and an air line was provided to externally cool the vessel when required. Any evaporation was controlled by a reflux condenser. The redox electrode was submerged in acid rather than being exposed to the offgas stream. Above $80^{\circ} \mathrm{C}$, the heat generated by dissolving 4 moles of uranium supplied enough heat to carry the reaction to the boiling point. Under these conditions, temperature was maintained by air cooling.

As shown in Table 3.2, the results are similar to those obtained with the smaller pot dissolving system. Run 1 consisted of a 45 minute baseline run with no process control. Runs 2 and 3 , which used peroxide with a redox control, indicate that it is possible to reduce emissions to approximately $1 \%$ of baseline. They also illustrate the major difficulty with redox control: the rate of uranium dissolution decreased substantially more than the decrease in available surface. 
Table 3.2. Large scale 4 inch diameter safe geometry dissolver tests ${ }^{a}$

\begin{tabular}{|c|c|c|c|c|c|c|c|c|}
\hline \multirow{2}{*}{ Run } & \multicolumn{2}{|c|}{ Uranium consumed } & \multirow{2}{*}{$\begin{array}{c}\mathrm{H}_{2} \mathrm{O}_{2} \\
\text { added } \\
\mathbf{m M}\end{array}$} & \multirow{2}{*}{$\begin{array}{c}\text { Initial } \\
\text { area } \\
\mathrm{cm}^{2}\end{array}$} & \multirow[b]{2}{*}{${ }^{\circ} \mathrm{C}$} & \multirow{2}{*}{$\begin{array}{c}\text { Time } \\
\mathbf{h}\end{array}$} & \multirow{2}{*}{$\begin{array}{l}\mathrm{NO}_{\mathrm{x}} \\
\mathrm{mM}\end{array}$} & \multirow{2}{*}{ Remarks } \\
\hline & g & $\mathbf{m} \mathbf{M}$ & & & & & & \\
\hline 1 & 142 & 0.60 & 0 & 398 & 99 & 0.75 & 1.11 & No peroxide \\
\hline 2 & 48 & 0.20 & 122 & 388 & 99 & 1.50 & 0.011 & Redox control \\
\hline 3 & 43 & 0.18 & 77 & 376 & 99 & 2.25 & 0.012 & Redox control \\
\hline
\end{tabular}

$a_{4} M, 952 \mathrm{~g}$ Uranium, $0.75 \mathrm{~h}$ time intervals.

\section{Experimental Difficulties}

This series of bench tests proceeded as planned with one major exception: peroxide pumping. Although the control scheme for this series of experiments has remained unchanged, the pumps and controllers for this series of experiments have undergone several changes. The control process is essentially to pump in peroxide whenever the redox reading passes a preset limit or whenever brown fumes appear. The first setup consisted of a syringe pump connected to an HP-85 controlled relay. The programming language was HP BASIC. The HP-85 monitored redox reading periodically. When the reading passed $900 \mathrm{mV}$, the relay powering the pump was turned on, and peroxide was added to the pot dissolver at a slow and controlled rate. When the reading passed below $850 \mathrm{mV}$, the pump was turned off.

The preliminary series of bench experiments showed that the control strategy was capable of providing adequate emissions control. However, the pumping setup had obvious problems. During a dissolving run, the pump was filled with slightly more than the predicted amount of peroxide. During the run, the peroxide gradually formed gas bubbles. Across a long run, the bubbles might displace almost all of the peroxide into the dissolver. When the pump was turned on near the end of a run, little or no peroxide was pushed into the dissolver.

After discussions with pump companies, we decided to try a small micrometering pump which was fitted with standard 1/4-28 NF threaded tubing connectors for use with low-volume narrow gauge tubing. We wanted a pump system in which only inert materials were in direct contact with peroxide. We also wanted a pump which was suitable for use in the scrap dissolving environment and which was designed expressly for use in computer or timer controlled systems. The small pump we chose has three individual solenoids which permit individual computer control of the stroke 
and timing of the inlet and outlet valves. The surfaces contacting peroxide are teflon, and the pump is capable of repeatedly dispensing up to 50 microliters per pump cycle. It offers low internal volume and well swept passages. Peroxide volumes ranging down to tens of microliters can be pumped in a single stroke, while the continuous pumping rate is between 300 and $400 \mathrm{ml}$ per $\mathrm{hr}$.

The control circuitry permits selection of both forward and reverse pumping, as well as variable pumping volumes, remotely. The pump can also be remotely opened to permit purging. Although the manufacturer indicates that the pump does not perrnit seepage during resting, we have not found this to be the case. The pump design features long-lived, chemically inert, Teflon diaphragms. As with many diaphragm pumps, it can be run dry without damage.

We also decided to continue tests with a modern control system using current computers since this would better reflect the type of system that could be bought to control tray dissolving processes. The best choice appeared to be a combination of Strawberry Tree internal personal computer cards, Workbench software with a graphical user interface, and analog and digital input-output. The boards, wiring, and software can be transferred between personal computers or duplicated in successive personal computers at a reasonable cost in a reasonable time period. The graphical user interface makes it simple for even untrained personnel to modify the basic control programs for the system. The systems can be used for data logging as well as control.

The computer control and pumping system did, however, have some problems which we are currently addressing. First, we found that, because of the dissolved oxygen in the hydrogen peroxide, positive pressure was required to prime the computer pump. Second, we found that experimentation was required to set the timing for the individual pump cylinders and solenoids. At a stroke rate of $0.33 \mathrm{~Hz}$, the pump provided acceptable $\mathrm{H}_{2} \mathrm{O}_{2}$ addition and redox control. Even with flooded suction, oxygen bubbles eventually formed in the intake line. This reduced the amount of liquid delivered per stroke and the pump output pressure.

Any pumping system designed for peroxide addition to the tray dissolvers should have flooded suction. Pump capacity should reflect the need to add a substantial fraction of the peroxide at the beginning of the run. Alternatively, manual peroxide addition at the start of a run could be included in startup procedures. 


\section{Resolution of Engineering Concerns}

This series of bench-scale dissolving tests was performed to determine whether an $\mathrm{NO}_{\mathrm{x}}$ control based on redox potential and scaleup strategy were feasible. Initial manually controlled experiments indicated that the control strategy was reasonably effective in controlling $\mathrm{NO}_{\mathrm{x}}$ offgas from batch dissolvers. Experiments to provide a basis for scaleup, selecting system components, materials of construction, and a process control strategy were then performed. The bases for selection, together with process recommendations, are discussed below.

\section{Electrodes}

Electrode construction. We wanted to select a redox electrode which would provide satisfactory service for periods of at least ten dissolver batch runs. In order to meet this goal, we decided to use industrial type electrodes, rather than less expensive (and less durable) lab models. As shown in Fig. 4.1, industrial electrodes typically consist of a glass electrode

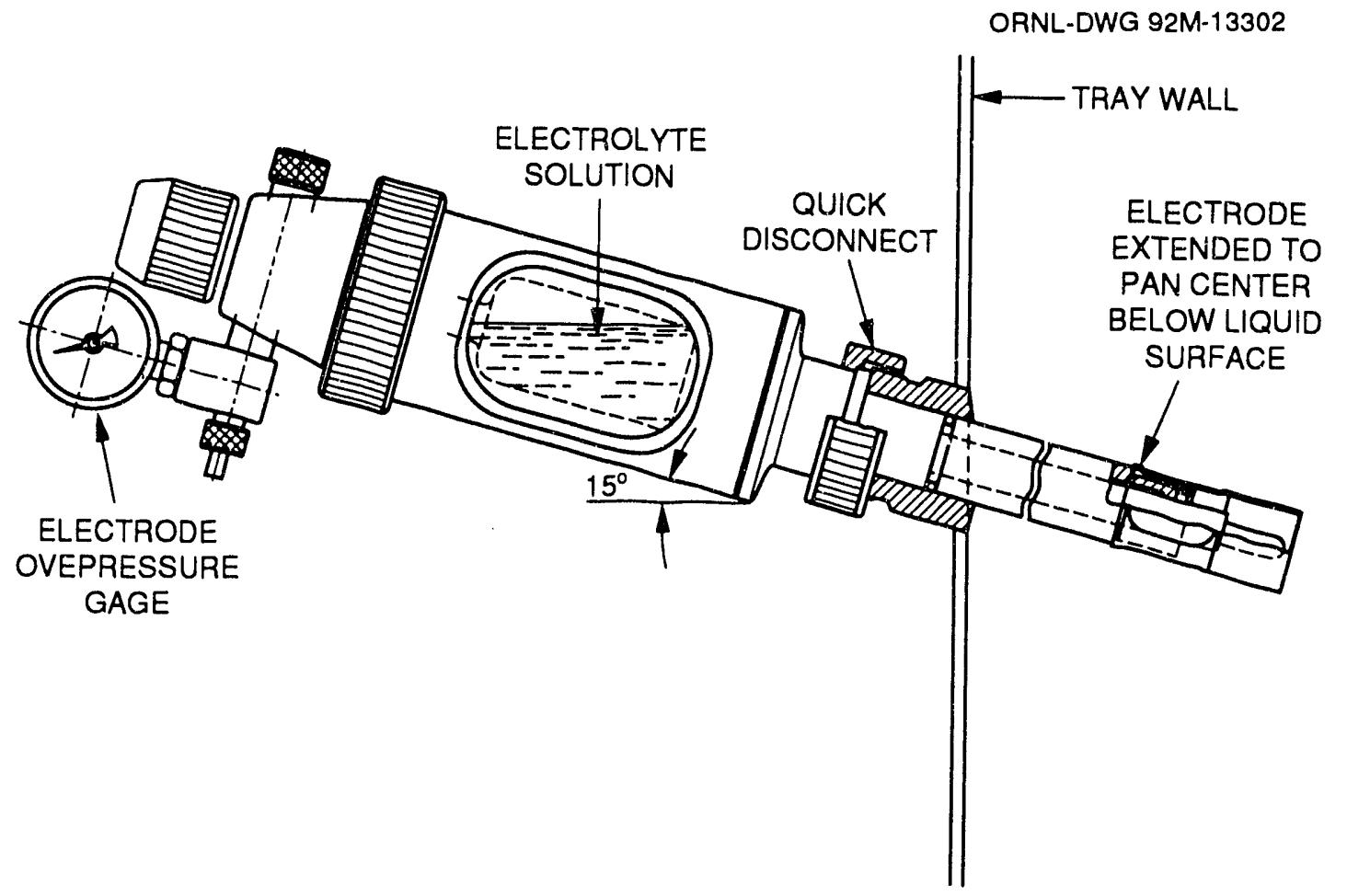

Fig. 4.1. Typical horizontally-mounted industrial electrode. 
inside a protective housing. The electrode can be installed either horizontally, as shown in the figure, or vertically, depending on the application. Electrode housing is constructed so that it provides physical protection for the glass portions of the electrode as well as a method for easily inserting the electrode into the process vessel. For liquid electrodes, the electrode filling solution is maintained under pressure to allow operation near the boiling point and to prevent backflcw into the electrolyte by providing a very small flow gradient through the electrode. Additionally, electrodes which use a potassium nitrate bridge, rather than bringing potassium chloride into direct contact with the solution, are available. These are desirable because they minimize contamination of dissolving solutions with chloride.

Changing the electrodes. Ingold and other industrial process control electrodes have been developed for extended life. This is, however, expected to be only a few weeks to a few months under the projected process conditions near $100^{\circ} \mathrm{U}$. To decrease problems, configurations which permit ropid electrode replacement were selected. The preferred configuration provides a protective housing which is inserted directly into a threaded socket welded into the dissolver pan housing. The housing, which is made of either PVDF or stainless steel, can be readily removed for cleaning or eiectrode replacement. The glass-body electrodes are supported and protected by the housing. Electrical connection is provided by standard, readily replaced, plug-in rables. The electrodes can be replaced by a chemical process operator in a few minutes.

Insertion. The redox electrodes work equally well when used in the liquid or in the gas. The electrodes can also be inserted vertically or at an angle ( $>15^{\circ}$ from horizontal) into the liquid. The electrode should be immersed at least a diameter into the liquid. Although all of these insertion methods are feasible from a measurement standpoint, they do not provide equivalent ease of use and replacement. For example, contact with a piece of metal scrap may change the redox reading if it touches the end of an electrode. The glass tip of the electrode can also be cracked or broken by impact with pieces of scrap metal. A vertical angle of insertion was selected to minimize these potential problems.

Metal or plastic. Materials of construction for the housing presented the first question to address in choosing an electrode. Durability, strength, and inertness were important because the housing would be required to last for periods of months to years in boiling nitric acid. The supplier we selected, Ingold, suggested that we evaluate PVC, PVDF, and stainless steel housing. Although the most available and inexpensive material, PVC is also the least durable. The materials durability charts provided by suppliers indicated that 
any of the three materials had a good probability of working. However, the high temperature used in dissolvers was likely to damage materials across a period of several weeks. We decided to test whether the PVC would survive by the simple expedient of taking a standard PVC fitting and boiling it in $30 \%$ nitric acid for $1,000 \mathrm{~h}$. At the end of the period, the fitting had changed dimension and color. It was obviously cracked and starting to disintegrate. We decided that stainless steel or PVDF housing, although more expensive, were likely to prove more satisfactory in operation.

Plug compatibility. A variety of plugs are used worldwide in $\mathrm{pH}$ and redox electrode cables. The U.S. standard which used two separate plugs has been in use here for decades; however, it is rapidly being replaced with BNC connectors which are used extensively with coaxial cables. The BNC connector is rugged and dependable. We recommend electrodes fitted with BNC cabling.

\section{Computerized Pump Control}

Micropump evaluation. Except for the need for flooded suction, the micropump performed reasonably well in bench tests. (The vapor lock caused by oxygen bubbles has been discussed in more detail in Chapter 3.) The pump was operated over a wide range of timing cycles and actuated using computer monitored redox setpoints. Careful control of the pump inlet and outlet valves was required to prevent flow through the pump when the inlet was pressurized and the pump was not actuated.

Feedback control loop. At the start of a run, large volumes of peroxide are used for initial redox control. Adding several liters of peroxide to a tray dissolver manually requires a few minutes. A pump-controller system is likely to take tens of minutes to add this material. We recommend manual addition of peroxide at any time, e. g. startup or high-volume acid replacement, where a liter or more of peroxide is likely to be needed by the tray dissolvers.

During a run, an automatic pump-controller system is more effective because it surveys and corrects redox in the dissolver every few seconds. This provides much better emissions control then does manual addition.

Electrode setpoints. Using a dead band of $50 \mathrm{mV}$ was generally satisfactory although some overshoot particularly on the low side was observed (especially using manual addition). A suitable setpoint and addition rate will keep this from causing a serious problem. 


\section{Peroxide and Nitric Acid Needs}

In closed dissolvers on uranium, nitric acid and peroxide are added in roughly equal amounts to maintain redox. In open pan dissolvers, more peroxide relative to nitric acid is used because the nitric acid concentration tends to increase with evaporation.

Accurate and consistent metering of peroxide is critical to successful emissions minimization. In order to do this, a reliable reagent dispensing system is required. As shown in our earlier tests, this can be accomplished in a variety of ways. An operator can manually add peroxide and nitric acid to maintain redox potential. This is perhaps the simplest and, in many ways, the most reliable, system but does use the largest amount of operator time.

With operator, setpoint, or computer control, a metering pump can be used to add nitric acid and peroxide. Although nitric acid addition is straightforward, peroxide addition presents an operational challenge. As noted earlier, the major problem in peroxide metering is the formation of bubbles in the lines. It is expected that this difficulty will be resolved by using a pumping system with flooded suction. Peroxide stabilization is also expected to mitigate this problem.

\section{Industrial No $\mathrm{x}_{\mathrm{x}}$ Measurement Methods}

Technology. $\mathrm{NO}_{\mathrm{x}}$ can be measured in a variety of ways. For our laboratory studies, we used a combination of caustic stripping and direct spectrophotometric measurement of light absorption.

The historically standard method for measuring $\mathrm{NO}$ and $\mathrm{NO}_{\mathrm{x}}$ in gaseous streams, including industrial stack gases, engine emissions, and process emissions, has been thermoluminescence. The gaseous stream is oxidized using ozone prior to measurement. The instruments used typically require frequent recalibration with a span gas; some current instruments run a continuous span gas calibration. Significant drift can occur within a few minutes due to a variety of factors, including gas pressure variations, condensation in the sampling lines, poor sample gas conditioning, particulate, and changes in gas temperature.

Changes in emissions management mandated by tie Clean Air Act have spurred the development of a variety of new $\mathrm{NO}_{\mathrm{x}}$ measurement technologies. Infrared measurement techniques, such as nondispersive infrared spectroscopy (NDIR), simplify maintenance and standardization procedures. For direct measurement of individual chemical species in 
offgases, a Fourier-Transform Infrared, or FTIR, system with fiber optic lightpipes has found industrial use. Changes in automotive exhaust management are also spurring the development of new, low-cost technologies which link output from metal oxide sensors directly to a computer-controller system.

Measurement of $\mathrm{NO}_{\mathrm{x}}$ is changing rapidly because of improvements in electronic and control devices. Every system has its benefits and its problems. When a measurement and control system is needed, uranium dissolving operations staff should review the available technology, consult researchers involved in developing rew instruments, and select the technology that, at the time of instaliation, appears to best suit their needs.

\section{System Configuration and Mechanics}

Mixing. The current dissolvers use a safe-geometry slab. Even in materials which are not density stratified, this configuration is usually characterized by poor mixing. Improving mixing is likely to increase the rate of metal dissolution. Several simple mechanical mixers have been used successfully in other systems. A magnetically-actuated flipper system used in stainless steel biological shakers seems likely to be effective in the tray dissolvers and should be tested.

\section{Scaleup to Production Vessels}

The bench tests discussed earlier support several generic observations which are likely to be relevant to scale-up tests and process equipment design. Ensuring the reliability and safety of dissolving systems for enriched uranium processes presents additional challenges. However, many of the basic design and control system problems are generic to the chemistry of uranium dissolving rather than a function of a single dissolving process or control system. Individual process considerations are discussed below.

Basic instrumentation needs for the various control methods. Several instrumentation needs are common to manual and automated processes. Temperature indication will help the operator in assessing the rate of reaction and will provide an early indication of changes in reaction rate at temperatures below the boiling point. Mixing to increase the rate of metal dissolution is also a fundamental process requirement. In tray dissolvers, gas bubbles provide predominantly vertical mixing; however, horizontal mixing may be needed to equalize the conditions over the tray. This will help to make instrumental measurements match average process conditions. 
Process quality assurance and operator efficiency will be increased by automatic data logging and automatic instrument monitoring systems. A chemical operator is responsible for several tray dissolvers. To monitor process history, the operator will be required to periodically log readings from several instruments during a dissolver run. Automatic logging and, as appropriate, process control instrumentation will decrease the amount of time that an operator has to spend logging. It will also make process data directly available to process staff and supervision.

Reagent addition methods. Peroxide bubbles form and block liquid flow in both manual and automatic systems. Excess line and pump capacity is required in both manual and automatic systems to compensate for bubbles. Any pumping system needs to reflect this problem by incorporating flooded suction and excess capacity in its design.

Equipment setup. The basic tray configuration needed are illustrated schematically in Fig. 4.2. Provision was made to permit insertion of the redox electrodes vertically and at an angle to investigate the best location. Provision to modify the vents and to provide a liquid seal on the tray overflow was also included.

Safety constraints. After safe geometry design, the major consideration is keeping peroxide concentration low. This is accomplished by a) limiting incoming peroxide and nitric acid streams to $30 \%$ concentration to prevent violent reactions and excessive peroxide decomposition and b) by keeping redox potential as high as feasible to lower excess dissolver peroxide concentration.

NO measurement. A number of methods for determining $\mathrm{NO}_{x}$ concentrations in air are available. First, operating personnel should be trained in visual $\mathrm{NO}_{\mathrm{x}}$ estimation as even the best chemical measurement fails occasionally. Even though quantitative instrumentation will be used, it would be prudent to develop and provide visual $\mathrm{NO}_{x}$ estimation procedures, including visual comparison standards. Along the same line, inexpensive hand-held single use chemical $\mathrm{NO}_{\mathrm{x}}$ estimation tubes are available. These permit estimation of $\mathrm{NO}_{x}$ concentration in air and will work when instruments are down. Instrumental measurement, which is convenient and readily added to a logging procedure, is presently expensive and involved. In selecting any system, care should be exercised to select instrumentation which can be readily calibrated and repaired. 


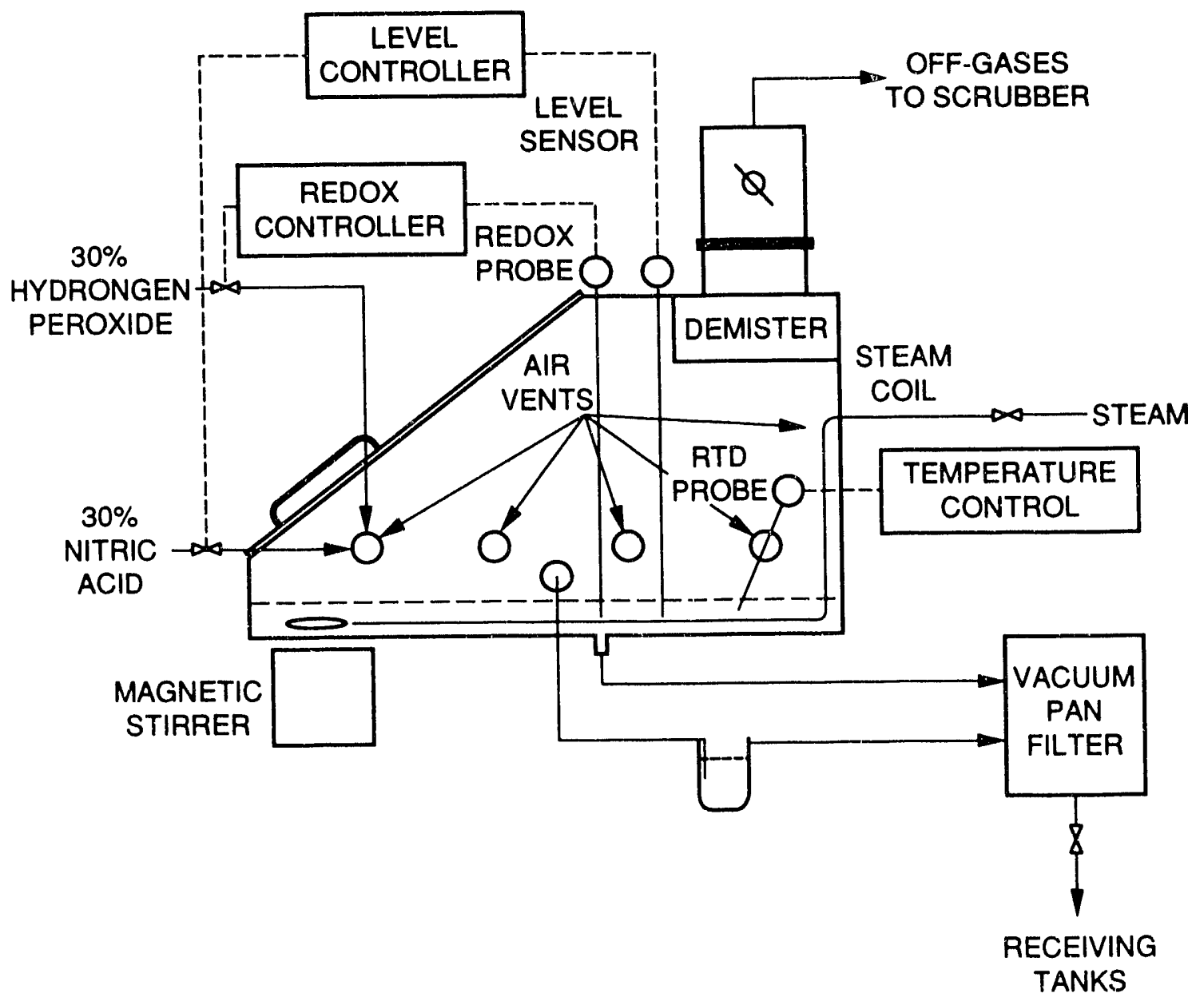

Fig. 4.2. Modified tray dissolver.

\section{Experimental Plan}

The experimental plan essentially involves starting with simple changes in the established process, evaluating the results, and gradually increasing $\mathrm{NO}_{\mathrm{x}}$ control measures. The main criterion in working toward a fully automated system is maintenance of the safe, reliable uranium dissolving process while process chemistry is fine-tuned to reduce emissions.

The first recommended tests involve modification of the tray dissolvers to support redox electrodes and improved horizontal mixing. A short test run using nitric acid and peroxide without uranium metal to test equipment setup is recommended prior to metal dissolving tests. This will ensure that both test and dissolving equipment are in working order and permit the 
operator to become familiar with the new equipment. When the new equipment is acceptable, the performances of plastic and stainless steel sheathed glass redox electrodes can be evaluated and compared.

Electrolyte selection is one of the major goals of this series of tests. There are several variables in electrode construction, placement, and filing electrolyte. The chloride concentrations in dissolving solutions obtained with the potassium nitrate bridge electrode should be compared with the chloride concentrations obtained with the other test electrodes to see if the more complicated bridge electrodes are worthwhile. This series of tests are expected to require several weeks for completion.

Outlines of the features likely to be incorporated in manual, continuous, and automatic peroxide addition to tray dissolvers are provided in Appendix A. Starting with the manual procedure and gradually increasing the use of automation is recommended. Detailed test protocol is expected to reflect knowledge gained during earlier tests and in-house procedures currently in development.

Our preliminary bench tests indicated that the safe-geometry cylindrical dissolver was feasible. If this unit could be developed into a prototype system, it has the potential for running larger dissolving batches on a continuous or semi-continuous basis. If the continuous or automatic tray dissolver tests indicate significant $\mathrm{NO}_{\mathrm{x}}$ reduction, further development of the cylindrical geometry system should be pursued for alloy and for oxide dissolving. 


\section{References}

Altshuller, A. P., and A. F. Wartburg. 1960. Ultraviolet determination of nitrogen dioxide as nitrite ion. Analytical Chemistry 32(2): 174-177.

Baker, F. B., and T. W. Newton. 1961. The reaction between uranium (IV) and hydrogen peroxide. Journal of Physical Chemistry 65: 1897-1899.

Blick, M.; Clucas, J.; McDonough, C.; and Woods, S. 1992. NO removal in the stainless steel pickling industry with hydrogen peroxide. In Chemical oxidation: technologies for the nineties, ed. W. W. Eckenfelder, A. R. Bowers, and J. A. Roth, pp. 78-88. Lancaster PA: Technomic Publishing Company, Inc.

Bresee, J. C.; Foster, D. L.; and Nurmi, E. O. 1956. Continuous dissolution of uraniumaluminum fuels. Oak Ridge National Laboratory report ORNL-1672.

Bresee, J. C.; Foster, D. L.; and Nurmi, E. O. 1959. Continuous dissolution of uraniumaluminum fuels in a trickle-type column dissolver. In Nuclear engineering - part $V$. American Institute of Chemical Engineers Chemical Engineering Progress Symposium Series 55(22): 25-32.

Caracciolo, V. P., and J. H. Owen. 1970. Electrolytic dissolution of power reactor fuel elements. In Progress in Nuclear Energy, Vol. 4, ed. C. E. Stevenson, E. A. Mason, and A. T. Gresky, pp. 81-119. New York: McGraw-Hill Book Company, Inc.

Carson, R. H.; Norris, R. D.; and Schellinger, R. 1982. The role of oxidizing agent in the chemistry of in-situ uranium leaching. Journal of Petroleum Technology 1982(4): 937-946.

Dalin, I. 1988. Method of reducing the emission of nitrogen oxides from a liquid containing nitric acid. European patent $0259533 \mathrm{~A} 1$.

Eligwe, C. A., and A. E. Torma. 1986. Advances in the hydrogen peroxide leaching of uranium ores. Metallwissenschaft Technik 40: 491-496.

Eligwe, C. A.; Torma, A. E.; and De Vries, F. W. 1982. Kinetics of uranium extraction from a New Mexico ore by sulfuric acid-hydrogen peroxide. Metallwissenschaft und Technik 36(2): 135-140.

Foster, D. L., and E. O. Nurmi. 1952. Dissolution of uranium-aluminum alloy slugs. Oak Ridge National Laboratory report ORNL 1105.

Foster, D. L.; Savolainen, J. C.; and Wymer, R. G. 1956. Nuclear reactor fuel dissolution. In Proceedings of the International Conference on the Peaceful Uses of Atomic Energy, Vol. 9, Reactor technology and chemical processing, ed. R. A. Charpie, et al., pp. 546-550. New York: United Nations. 
Gens, T. A., and W. E. Clark. 1959. Laboratory development of the Perflex Process for dissolution of uranium alloy fuels in hydrofluoric acid - hydrogen peroxide solutions. Oak Ridge National Laboratory report ORNL-CF 59-11-23.

Hatcher, W. H., and D. W. Maclauchlan. 1938. Conductivity data of aqueous mixtures of hydrogen peroxide and nitric acid. Canadian Journal of Research, Section $B$ 16(8): 253-259.

Hofmann, J.; Holtz, H.; and Wagner, G. 1979, Process and apparatus for producing nitrogen oxide-free waste gases during the dissolving of irradiated fuel elements. West German patent 2,751,1235.2-41.

Hufner, E. H. R. 1984. Modular design of a reprocessing plant dissolver off-gas system. In Radioactive Waste Management, pp. 455-473. International Atomic Energy Agency report IAEA-CN-43/447.

Huygen, C. 1970. Reaction of nitrogen dioxide with Griess type reagents. Analytical Chemistry 42(3): 407-409.

Kemperman, D. R. 1976. Metal dissolution process. U. S. patent $3,945,865$.

Martin, F. S., and G. L. Miles. 1958. Chemical processing of nuclear fuels. New York: Acadernic Press, Inc.

Miles, G. L. 1955. The fumeless dissolving of uranium. A. E. R. E. Harwell report C/R 1804, declassified from C/R 298 (1949).

Miles, G. L.. 1956. The fumeless dissolving of uranium. In Progress in Nuclear Energy, ed. F. R. Bruce, J. M. Fletcher, H. H. Hyman, and J. J. Katz, pp. 97-101. New York: McGraw-Hill Book Company, Inc.

Schortmann, W. E., and DeSesa, M. A. 1958. Kinetics of the dissolution of uranium dioxide in carbonate-bicarbonate solutions. In Proceedings of the International Conference on the Peaceful Uses of Atomic Energy, Vol. 3, Processing of Raw Materials, ed. R. A. Charpie, et al., pp. 333-341. New York: United Nations.

Schumb, W. C.; Satterfield, C. N.; and Wentworth, R. L. 1955. Hydrogen peroxide. American Chemical Society monograph 128. New York: Reinhold Publishing Corporation.

Selby, G. W., and R. M. Counce. 1988. Aqueous scrubbing of dilute nitrogen oxide gas mixtures. Industrial and Engineering Chemistry Research 27(10): 1917-1922.

Swanson, J. L.; Bray, L. A.; Kjarmo, H. E.; Ryan, J. L.; Matsuzaki, C. L.; Pitman, S. G.; and Haberman, J. H. 1985. Laboratory studies of shear/leach processing of Zircaloy clad metallic uranium reactor fuel. Pacific Northwest Laboratory report PNL-5708.

Wymer, R. G., and R. E. Blanco. 1957. Uranium-aluminum alloy dissolution. Industrial and Engineering Chemistry 49(1): 59-61. 
Wymer, R. G., and D. L. Foster. 1956. Nuclear reactor fuel dissolution. In Progress in Nuclear Energy, ed. F. R. Bruce, J. M. Fletcher, H. H. Hyman, and J. J. Katz, pp. 85-96. New York: McGraw-Hill Book Company, Inc.

Wymer, R. G.; Helton, D. M.; Kibbey, A. H.; and Land, J. L. 1955. Caustic dissolution: preliminary studies. Oak Ridge National Laboratory report ORNL-1782.

Zhukovskii, Y. M.; Luksha, O. P.; Nenarokomov, E. A.; Pogorelyi, A. A.; and Pronin, A. V. 1987. Development of a mathematical model for the dissolution of uranium dioxide. I. Mathematical model for the dissolution of powdered uranium dioxide in nitric acid. Radiokhimiya 29(4): 500-505. 


\section{Appendix A. Proposed Changes to Tray Dissolver System - Scrap Recovery Procedure 50-37-90-112}

Several changes to Procedure 50-37-90-112 are proposed to accommodate the use of redox potential-based control systems. The changes include the calibration and use of redox electrodes, manual and automatic peroxide addition systems, and automated process control procedures. The tray dissolver procedures are presently in review and it is expected that changes covering these recommendations will be included in the new procedures. The changes cover Sections VI.E.2.d and VI.E.2.e.

\section{Original procedure:}

d. Add chemicals to tray dissolver as per parameters for dissolution of uranium alloy scrap materials.

e. Open steam and condensate valves for the appropriate tray dissolver (D-700, D-800, D-900) and bring solution to sub-boiling temperature.

\section{Manual addition of peroxide, proposed procedure:}

d(i). Fill a $10 \mathrm{~L}$ safe bottle with $9 \mathrm{~L}$ of $30 \%$ hydrogen peroxide. Move bottle to the holder at the hood and secure.

d(ii). Fill the tray with $30 \%$ nitric acid until it covers the scrap metal (9 to $18 \mathrm{~L}, 2.5$ to $5 \mathrm{~cm}$ deep).

d(iii). Calibrate and install the redox electrode-pH meter.

e(i). Open steam and condensate valves for the tray (D-700, D-800, or D-900).

e(ii). When solution temperature the system reaches 60 to $65^{\circ} \mathrm{C}$, begin adding small amounts of peroxide, usually $100 \mathrm{ml}$ or less, to the tray. When brown fumes are observed (i.e. when the redox potential exceeds $1025 \mathrm{mV}$ ) add $100 \mathrm{ml}$ portions of peroxide. Do not exceed a total of $1000 \mathrm{ml}$ of $30 \%$ hydrogen peroxide during tray warm-up without 
notifying your supervisor. Permit tray temperature to continue rising until it reaches about 85 to $90^{\circ} \mathrm{C}$, and then throttle the steam value to prevent overheating. To ensure proper operation, check the redox reading every ten minutes initially. As the run progresses, the time between readings can be lengthened (or shortened) as needed.

WARNING - Never add peroxide to a tray if there is any sign of organics floating on the surface of the solution in a tray or if the scrap metal is covered with organics. Certain organics react violently with peroxide.

Note: Do not overheat as this may cause excessive nitric acid evaporation and decomposition of hydrogen peroxide.

Note: When hydrogen peroxide addition starts, there is a one-time increase in redox potential, typically on the order of $100 \mathrm{mV}$. The redox increases sharply during the first addition of peroxide and then decreases to expected control levels.

Note: If the redox potential is below $800 \mathrm{mV}$, do not add more peroxide. The preferred operating range is $1025 \mathrm{mV}$ to $1050 \mathrm{mV}$ although there will be some variability in redox readings given different electrodes. Therefore, redox values given are to be regarded as approximate. Redox potentials below $700 \mathrm{mV}$ may cause dissolved uranium to precipitate as white uranium peroxide. This can be prevented by adding hydrogen peroxide more slowly. The operator should discontinue adding peroxide until the redox potential increases above $900 \mathrm{mV}$ and the precipitate dissolves. If the precipitate does not dissolve in an hour, the operator should either stir the solution or add a small amount of fresh nitric acid.

Caution: Peroxide decomposes into oxygen and water, so add slowly to avoid violent reaction. Fumeless operation is indicated by bright yellow color in tray solutions and the gentle bubbling of oxygen around the scrap metal.

e(iii). Carefully add $100 \mathrm{ml}$ portions of $30 \%$ hydrogen peroxide to trays as needed to control fumes. Do add more than $750 \mathrm{ml}$ of peroxide per half hour without the approval of your supervisor.

Note: A repipet or other hand pump can be used to control peroxide addition and minimize the potential for spills.

Note: If the color in the dissolver liquid changes from bright yellow to orange or if any brown $\mathrm{NO}_{\mathrm{x}}$ fumes are observed in the vapors over 
solutions in the tray, the operator may add an additional portion of peroxide.

e(iv). If the peroxide decomposition rate appears to be higher than usual, add fresh nitric acid to the tray to dilute it.

e(v). Check hydrogen peroxide supply and replenish as needed.

Note: Do not exceed $27 \mathrm{~L}$ per shift (three $10 \mathrm{~L}$ safe bottles) without approval by supervision.

\section{Continuous addition of peroxide, proposed procedure:}

d(i). Fill a $10 \mathrm{~L}$ safe bottle with $9 \mathrm{~L}$ of $30 \%$ hydrogen peroxide. Move bottle to the holder at the hood and secure.

d(ii). Place the suction line for the peroxide pump in the bottom of the peroxide-filled safe bottle. Be sure that the tubing reaches the bottom of the bottle.

d(iii). Put the stirrer bar in the tray and test it.

$d$ (iv). Turn on the peroxide pump for 1 minute and verify that it is dispensing peroxide into the tray without leaking. If leaks occur, turn off the peroxide pump and report it to the supervisor.

$d(v)$. Open the manual nitric acid valve, turn on the nitric acid feed system, and observe it fill the tray with $30 \%$ nitric acid until it covers the scrap metal (9 to $18 \mathrm{~L}, 2.5-5 \mathrm{~cm}$ deep). If the nitric acid doesn't automatically shut off when the tray is full ( $4 \mathrm{~cm}$ deep), close the manual nitric acid valve, notify the supervisor, and feed acid manually as needed. Turn on the stirrer.

d(vi). Calibrate and install the redox electrode-pH meter.

e(i). Open steam and condensate valves for the tray (D-700, D-800, or D-900).

e(ii). When the system reaches 60 to $65^{\circ} \mathrm{C}$, start the peroxide feed system, and allow to tray to heat to 85 to $90^{\circ} \mathrm{C}$. Either throttle the steam valve to prevent overheating once the desired operating temperature is reached or turn on the automatic temperature controller. 
WARNING - Never add peroxide to a tray if there is any sign of organics floating on the surface of the solution in a tray or if the scrap metal is covered with organics. Certain organics react violently with peroxide.

Note: Do not overheat as this may cause excessive nitric acid evaporation and decomposition of hydrogen peroxide.

Note: When hydrogen peroxide addition starts, there is a one-time increase in redox potential, typically on the order of $100 \mathrm{mV}$. The redox increases sharply during the first addition of peroxide and then decreases to expected control levels.

e(iii). Until experience deems otherwise, adjust the peroxide addition rate by manually adjusting the pump speed to keep the tray redox potential between 1025 and $1050 \mathrm{mV}$.

Note: Measure the redox potential of the tray solution. If the redox potential exceeds $1075 \mathrm{mV}$ or if brown fumes appear, increase the pump speed. If the redox potential drops below $800 \mathrm{mV}$, decrease the pump speed. If precipitation occurs or the redox potential drops below $650 \mathrm{mV}$, stop the pump until the redox potential returns to the operating range and then adjust the pump speed to a slower setting.

Note: There may be some variability in readings between individual electrodes.

Note: To ensure proper operation, check the redox reading and the pump rate every ten minutes initially. As the run progresses, the time between readings can be lengthened (or shortened) as needed.

Note: If the pump will not prime due to vapor lock, briefly turn the valve on the vacuum for the peroxide suction bottle to fill the pump and remove air from the feed line.

Note: Redox potentials below $700 \mathrm{mV}$ may cause dissolved uranium to precipitate as white uranium peroxide. This can be prevented by slower addition of hydrogen peroxide. The operator should discontinue adding peroxide until the redox potential increases above $900 \mathrm{mV}$ and the precipitate dissolves. If the precipitate does not dissolve in a few minutes, the operator should either stir the solution or add a small amount of fresh nitric acid.

Note: If the color in the dissolver liquid changes from bright yellow to orange or if any brown $\mathrm{NO}_{\mathrm{x}}$ fumes are observed in the vapors over 
solutions in the tray, the operator may manually add an additional 100 $\mathrm{ml}$ aliquot of peroxide or increase pump speed briefly until the fumes disappear.

Caution: Peroxide decomposes into oxygen and water so add slowly to avoid violent reaction. Fumeless operation is indicated by bright yellow color in tray solutions and gentle bubbling of oxygen around the scrap metal.

e(iv). If the peroxide decomposition rate appears to be higher than usual, add fresh nitrir acid to the tray to dilute it.

e(v). Check hydrogen peroxide supply every hour and replenish as needed. Do not exceed $27 \mathrm{~L}$ per shift (three $10 \mathrm{~L}$ safe bottles) without approval by supervision.

Note: As detailed in the calitration procedure, there is some variability in redox readings by a single electrode. The values given are to be regarded as approximate.

Note: If required, tray overflowing should be avoided by draining the tray and refilling with fresh acid and peroxide.

\section{Automatic addition of peroxide, proposed procedure:}

d(i). Fill a $10 \mathrm{~L}$ safe bottle with $9 \mathrm{~L}$ of $30 \%$ hydrogen peroxide. Move bottle to the holder at the hood and secure.

d(ii). Place the suction line for the peroxide pump in the bottom of the peroxide-filled safe bottle. Be sure that the tubing reaches the bottom of the bottle.

d(iii). Put the stirrer bar in the tray and test it.

d(iv). Turn on the peroxide pump for 1 minute and verify that it is dispensing peroxide into the tray without leaking. If leaks occur, turn off the peroxide pump and report it to the supervisor.

$d(v)$. Open the manual nitric acid valve, turn on the nitric acid feed system, and observe it fill the tray with $30 \%$ nitric acid until it covers the scrap metal (9 to $18 \mathrm{~L}, 2.5-5 \mathrm{~cm}$ deep). If the nitric acid doesn't automatically shut off when the tray is full ( $4 \mathrm{~cm}$ deep), close the manual nitric acid valve, notify the supervisor, and feed acid manually as needed. Turn on the stirrer. 
$\mathrm{d}($ vi). Calibrate and install the redox electrode-pH meter.

e(i). Open steam and condensate valves for the tray (D-700, D-800, or D900).

e(ii). When the system reaches 60 to $65^{\circ} \mathrm{C}$, turn on the automatic peroxide feed controller and peroxide pump, and allow to tray to heat to 85 to $90^{\circ} \mathrm{r}$. If the controller doesn't maintain the tray redox around 1025 to $1050 \mathrm{mV}$ then turn off the peroxide controller, notify your supervisor and revert to either continuous or manual feeding of peroxide. Either throttle the steam valve to prevent overheating once the desired operating temperature is reached or turn on the automatic temperature controller.

WARNING - Never add peroxide to a tray if there is any sign of organics floating on the surface of the solution in a tray or if the scrap metal is covered with organics. Certain organics react violently with peroxide.

No:e: Do not overheat as this may cause excessive nitric acid evaporation and decomposition of hydrogen peroxide.

Note: When hydrogen peroxide addition starts, there is a one-time increase in redox potential, typically on the order of $100 \mathrm{mV}$. The redox increases sharply during the first addition of peroxide and then decreases to expected control levels.

Note: Measure the redox potential of the tray solution. If the redox potential exceeds $1075 \mathrm{mV}$ or if brown fumes appear, verify the control system is working and, if so, increase the pump speed. If the redox potential drops below $800 \mathrm{mV}$, again check that the control system is working and, if so, decrease the pump speed. If the control system fails switch to continuous peroxide feed operation. If precipitation occurs or the redox potential drops below $650 \mathrm{mV}$, stop the pump until the redox potential returns to the operating range and then adjust the pump speed to a slower setting.

Note: There may be some variability in readings between individual electrodes.

Note: To ensure proper operation, check the redox reading and the pump rate every ten minutes initially. As the run progresses, the time between readings can be lengthened (or shortened) as needed. 
Note: if the pump will not prime due to vapor lock, briefly turn the valve on the vacuum for the peroxide suction bottle to fill the pump and remove air from the feed line.

Note: Redox potentials below $700 \mathrm{mV}$ may cause dissolved uranium to precipitate as white uranium peroxide. This can be prevented by slower addition of hydrogen peroxide. The operator should discontinue adding peroxide until the redox potential increases above $900 \mathrm{mV}$ and the precipitate dissolves. If the precipitate does not dissolve in a few minutes, the operator should either stir the solution or add a small amount of fresh nitric acid.

Note: If the color in the dissolver liquid changes from bright yellow to orange or if any brown $\mathrm{NO}_{\mathrm{x}}$ fumes are observed in the vapors over solutions in the tray, the operator may manually add an additional 100 $\mathrm{ml}$ aliquot of peroxide or increase pump speed briefly until the fumes disappear.

Caution: Peroxide decomposes into oxygen and water so add slowly to avoid violent reaction. Fumeless operation is indicated by bright yellow color in tray solutions and gentle bubbling of oxygen around the scrap metal.

e(iii). If the peroxide decomposition rate appears to be higher than usual, add fresh nitric acid to the tray to dilute it.

e(iv). Check hydrogen peroxide supply every hour and replenish as needed. Do not exceed $27 \mathrm{~L}$ per shift (three $10 \mathrm{~L}$ safe bottles) without approval by supervision.

Note: As detailed in the calibration procedure, there is some variability in redox readings by a single electrode. The values given are to be regarded as approximate.

Note: If required, tray overflowing should be avoided by draining the tray and refilling with fresh acid and peroxide. 

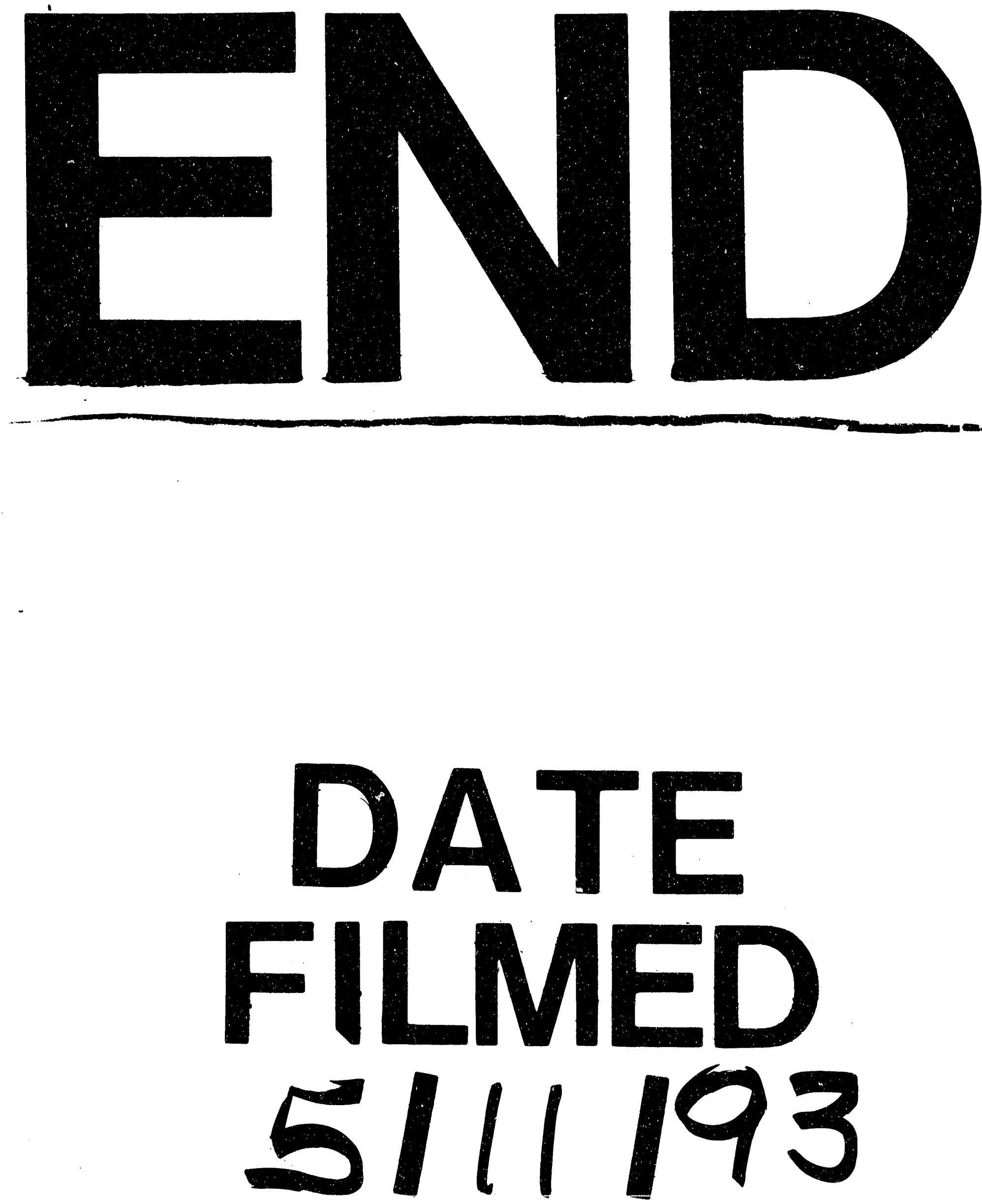
1 